\title{
Optimum Unambiguous Discrimination of Linearly Independent Pure States
}

\author{
Shengshi Pang ${ }^{1}$ and Shengjun $\mathrm{Wu}^{1,2}$ \\ ${ }^{1}$ Hefei National Laboratory for Physical Sciences at Microscale, \\ University of Science and Technology of China, Hefei, Anhui 230026, China \\ ${ }^{2}$ Lundbeck Foundation Theoretical Center for Quantum System Research, \\ Department of Physics and Astronomy, Aarhus University, DK-8000 Aarhus C, Denmark
}

(Dated: June 17, 2018)

\begin{abstract}
Given $n$ linearly independent pure states and their prior probabilities, we study the optimum unambiguous state discrimination problem. We derive the conditions for the optimum measurement strategy to achieve the maximum average success probability, and establish two sets of new equations that must be satisfied by the optimum solution in different situations. We also provide the detailed steps to find the optimum measurement strategy. The method and results we obtain are given a geometrical illustration with a numerical example. Furthermore, using these new equations, we derive a formula which shows a clear analytical relation between the optimum solution and the $n$ states to be discriminated. We also solve a generalized equal-probability measurement problem analytically. Finally, as another application of our result, the unambiguous discrimination problem of three pure states is studied in detail and analytical solutions are obtained for some interesting cases.
\end{abstract}

PACS numbers: 03.67.-a, 03.65.Wj, 03.65.Ta, 42.50.Dv

Keywords: Unambiguous state discrimination, optimum measurement strategy

\section{INTRODUCTION}

Discrimination of quantum states has been an interesting and attractive problem in quantum information science for a relatively long time [1, 2, 3]. Since deterministic and error-free discrimination of an arbitrary set of quantum states is generally impossible due to the basic principles of quantum mechanics, just like other "no-go" theorems [4, [5, [6], the problem of finding an effective scheme of state discrimination has attracted a lot of attention and has played an import role in the study of quantum communication and cryptography. Considerable work has been devoted to this problem and it has developed rapidly recently.

The task of state discrimination is to discriminate the state of a quantum system from a given finite set of possible states with certain prior probabilities, and there are mainly two kinds of strategies to complete this task. One kind of strategy is called minimum error discrimination [3, 7, 8] which requires that the average probability of identifying a wrong state is minimized. There have been numerous results for this kind of discrimination strategy $9,10,11,12,13$, 14, 15, 16], some of which are quite interesting, like the weighted square-root measurement [17, 18, 19, 20, 21, 22, 23, 24] and that measurement sometimes does not aid in discriminating certain set of states [25, 26].

Another important kind of discrimination strategy is unambiguous discrimination pioneered by [27, 28, 29], which requires that no error occurs in the identification of the states at the expense of obtaining an inclusive result with some non-zero probability. A lot of research has also been performed on this kind of discrimination strategy [30, 31, 32, 33, 34, 35, 36, 37, 38], including unambiguous discrimination of symmetric states [39] and unambiguous discrimination between mixed states [40, 41, 42, 43, 44, 45, 46, 47, 48, 49.].

In addition to the above two strategies, research has also been performed on mixed strategies involving minimumerror discrimination and unambiguous discrimination together [50, 51, 52, 53], in order to achieve balance between the accuracy and the efficiency of state identification. It is, in general, very difficult to maximize the average probability of successfully discriminating the given states analytically, but some special techniques such as semidefinite programming have been employed to solve this computation problem numerically [38, 54, 55, ,56].

In this article we shall study the properties of the optimum strategy for unambiguous discrimination of $n$ pure states, and give a detailed method to obtain such an optimum strategy. According to [31, 32], one can manage to discriminate a set of states unambiguously with non-zero success probabilities if and only if the given states are linearly independent. We shall hold this assumption throughout this article. The main method we use for studying this problem is to put the individual probabilities that each state is successfully identified together as a vector in the $n$-dimensional real space $\mathbb{R}^{n}$ (and do the same to the $n$ prior probabilities), and study the properties of the minimum eigenvalue of the matrix $X-\Gamma$ under the optimum strategy by vector analysis techniques $(X$ is the Gram matrix of the $n$ states to be discriminated and $\Gamma$ is the diagonal matrix with the success probabilities as its diagonal elements, they will be defined explicitly later in Theorem 10. From the properties of the minimum eigenvalue of $X-\Gamma$, we shall establish two sets of equations that the optimum solution of the unambiguous discrimination problem must satisfy in different situations, and these equations will turn out to have some intuitive geometrical meanings. 
This article is organized as follows. In Sec. II we shall give a general description of the unambiguous state discrimination problem that we are interested in and the POVM formalism that we shall use in this article. In Sec. III. we derive the properties of the optimum measurement strategy and the new equations that can be used to work out the optimum solution. Examples are also given to geometrically and numerically illustrate our method of solving the unambiguous discrimination problem. Sec. IV] is devoted to deriving an analytical formula which characterizes a simple relation between the maximum average success probability and the $n$ pure states to be unambiguously discriminated. In Sec. V] a generalized version of equal-probability-measurement (EPM) problem [56] is studied and an analytical solution is obtained. Finally we apply the results obtained in Sec. III to the case of three linearly independent pure states in Sec. VI and work out analytical solutions for some interesting cases.

\section{PROBLEM DESCRIPTION AND POVM FORMALISM}

In this article, our problem is how to unambiguously identify the state of a quantum system with the maximum average success probability since the discrimination of non-orthogonal states is generally probabilistic. And all we know is that this state belongs to a given set of $n$ linearly independent states $\left\{\left|\psi_{i}\right\rangle\right\}_{i=1}^{n}$ with given prior probabilities $\gamma_{i}(i=1, \cdots, n)$. We would like to obtain some analytical conditions that the optimum solution should satisfy, and provide a detailed method for obtaining the optimum solution of the problem.

In the following study of the problem, the $n$ prior probabilities will be denoted by a real vector $\gamma$ in the space $\mathbb{R}^{n}$ and the success probabilities $p_{i}(i=1, \cdots, n)$ for unambiguous outcomes will also be denoted as a real vector $\boldsymbol{p}$ in $\mathbb{R}^{n}$ for short. The average success probability for unambiguous discrimination can be written as $\bar{p}=\sum_{i=1}^{n} \gamma_{i} p_{i}=\gamma \cdot \boldsymbol{p}$ then.

Since only the probabilities of the measurement outputs are concerned in this problem, we shall use the POVM (positive-operator-valued measure) formalism [57] which is a good description for the statistics of a general physics process. A POVM consists of a set of POVM elements $\left\{\Pi_{i}\right\}$ satisfying $\Pi_{i} \geq 0$ and $\sum_{i} \Pi_{i}=I$, where $I$ represents the identity operator (or matrix). A Hermitian operator is said to be positive and denoted by " $\geq 0$ " if all of its eigenvalues are non-negative. To unambiguously identify the state, we require that

$$
p(j \mid i)=\left\langle\psi_{i}\left|\Pi_{j}\right| \psi_{i}\right\rangle=p_{i} \delta_{i j}, \forall i, j=1, \cdots, n
$$

where $p(j \mid i)$ represents the probability of obtaining the result $j$ when the original state of the system is actually $\left|\psi_{i}\right\rangle$ and $p_{i}$ denotes the probability of correctly identifying $\left|\psi_{i}\right\rangle$.

Let $\mathcal{H}$ denote the $n$-dimensional Hilbert space spanned by the given set of linearly independent pure states $\left\{\left|\psi_{i}\right\rangle\right\}_{i=1}^{n}$. The total Hilbert space of the system, denoted by $\mathcal{H}_{\text {total }}=\mathcal{H} \oplus \mathcal{H}^{\perp}$, may be larger than $\mathcal{H}$, where $\mathcal{H}^{\perp}$ denotes the subspace orthogonal to $\mathcal{H}$. Any operator acting on $\mathcal{H}_{\text {total }}$ is equivalent to an operator on $\mathcal{H}$ by projecting onto the space $\mathcal{H}$ when only the effect on $\mathcal{H}$ is considered. Therefore, without loss of generality, we shall restrict our POVM elements to those acting on the space $\mathcal{H}$ spanned by the given set of states. In order to discriminate the states $\left\{\left|\psi_{i}\right\rangle\right\}_{i=1}^{n}$ unambiguously, the POVM element $\Pi_{i}$ that identifies the $i$ th state $\left|\psi_{i}\right\rangle$ must be orthogonal to the subspace spanned by the other $n-1$ states according to Eq. (11), therefore the rank of $\Pi_{i}$ should be no larger than 1 , for all $i=1, \cdots, n$. Thus each POVM element that successfully identifies a certain state should have the form [38, 56]

$$
\Pi_{i}=p_{i}\left|\widetilde{\psi}_{i}\right\rangle\left\langle\widetilde{\psi}_{i}\right|
$$

where $p_{i}$ is the success probability to identify the $i$ th state and $\left|\widetilde{\psi}_{i}\right\rangle$ is an unnormalized state orthogonal to $\left|\psi_{j}\right\rangle$ for all $j \neq i$. It can be seen by substituting Eq. (2) into (1) that $\left|\left\langle\psi_{i} \mid \widetilde{\psi}_{i}\right\rangle\right|^{2}=1$ and there is freedom for each $\left|\widetilde{\psi}_{i}\right\rangle$ to have an arbitrary phase, and without loss of generality we make a specific choice of the phase such that

$$
\left\langle\psi_{j} \mid \widetilde{\psi}_{i}\right\rangle=\delta_{i j}
$$

for convenience.

Let $\Phi$ denote the matrix with $\left|\psi_{i}\right\rangle$ as its $i$ th column. Define $\widetilde{\Phi}$ as

$$
\widetilde{\Phi}=\Phi\left(\Phi^{\dagger} \Phi\right)^{-1}
$$

then

$$
\Phi^{\dagger} \widetilde{\Phi}=I
$$

Note that Eq. (4) cannot be simplified to $\widetilde{\Phi}=\left(\Phi^{\dagger}\right)^{-1}$ in general, because the states $\left\{\left|\psi_{i}\right\rangle\right\}_{i=1}^{n}$ may belong to a larger Hilbert space of which the dimension is greater than $n$, implying that the matrix $\Phi$ may not be a square matrix. (In 
the last paragraph, we only restrict the POVM elements to be those that act on the space spanned by these states, but we do not make any restrictions on the representation of these states.) Comparing (3) and (5), we know that $\left|\widetilde{\psi}_{i}\right\rangle$ is exactly given by the $i$ th column of the matrix $\widetilde{\Phi}$.

Since unambiguous discrimination of the states $\left\{\left|\psi_{i}\right\rangle\right\}_{i=1}^{n}$ is probabilistic if they are not orthogonal to each other, there exists a POVM element $\Pi_{0}$ which gives the inconclusive result, and it can be written as

$$
\Pi_{0}=I-\sum_{i=1}^{n} p_{i}\left|\widetilde{\psi}_{i}\right\rangle\left\langle\widetilde{\psi}_{i}\right| .
$$

Since any POVM element must be positive to represent a physically realizable process, it is required that

$$
\Pi_{0}=I-\sum_{i=1}^{n} p_{i}\left|\widetilde{\psi}_{i}\right\rangle\left\langle\widetilde{\psi}_{i}\right| \geq 0
$$

This positivity inequality is an essential constraint on the unambiguous discrimination scheme and is the starting point of the discussions in this article.

When the set of states $\left\{\left|\psi_{i}\right\rangle\right\}_{i=1}^{n}$ and prior probabilities $\gamma_{i}(i=1, \cdots, n)$ are given, the POVM elements for the measurement $\left(\Pi_{i}=p_{i}\left|\widetilde{\psi}_{i}\right\rangle\left\langle\widetilde{\psi}_{i}\right|, \Pi_{0}=I-\sum_{i=1}^{n} \Pi_{i}\right)$ depend only on the variables $p_{i}$, i.e. the success probabilities, since $\left|\widetilde{\psi}_{i}\right\rangle(i=1, \cdots, n)$ can be determined and explicitly given by the $i$ th column of $\widetilde{\Phi}$ defined in (4). Therefore, searching for the optimum solution is to find a set of success probabilities $p_{i}$ (corresponding to a point or a vector $\boldsymbol{p}$ in $\mathbb{R}^{n}$ ) such that their weighted average (with the prior probabilities $\gamma_{i}$ as the weights) is maximized under the restrictions $p_{i} \geq 0$ $(i=1, \cdots, n)$ and $\Pi_{0} \geq 0$. The optimum solution is denoted by $\boldsymbol{p}_{\text {opt }}$, and shall be called optimum point sometimes throughout the rest of the article; and the main goal of this article is to find the optimum point $\boldsymbol{p}_{\text {opt }}$.

\section{EXPLORATION OF THE OPTIMUM STRATEGY}

In this section, we shall obtain some properties of the optimum strategy for the unambiguous state discrimination problem, and provide a systematic way to obtain the maximum average success probability and the optimum measurement strategy.

\section{A. General properties and methods}

As the first step to study the optimum unambiguous state discrimination problem described in Sec. II, we are going to re-derive the positivity condition given by Duan and Guo [32] in a more concise way, using the POVM representation, and prove a convexity property of the set of all feasible $\boldsymbol{p}$ 's.

Theorem 1(Positivity and Convexity). Suppose $\left\{\left|\psi_{i}\right\rangle\right\}_{i=1}^{n}$ is a set of linearly independent pure states. Let $X=\Phi^{\dagger} \Phi$ where $\Phi$ is the matrix whose $i$ th column is $\left|\psi_{i}\right\rangle(i=1, \cdots n)$, and $\Gamma=\operatorname{diag}\left(p_{1}, \cdots, p_{n}\right)$ where $p_{i}$ is the success probability to unambiguously discriminate $\left|\psi_{i}\right\rangle(i=1, \cdots, n)$. Then i)

$$
X-\Gamma \geq 0, \quad \Gamma \geq 0 .
$$

ii) Let $\mathcal{S}$ denote the set of points $\boldsymbol{p}$ satisfying the positivity condition (8), then $\mathcal{S}$ is convex.

Proof. i) Using the definition of $\Gamma$ in this theorem, Eq. (7) can be re-written as

$$
I-\widetilde{\Phi} \Gamma \widetilde{\Phi}^{\dagger} \geq 0 .
$$

Substituting Eq. (4) into (9), we have

$$
I-\Phi\left(\Phi^{\dagger} \Phi\right)^{-1} \Gamma\left(\Phi^{\dagger} \Phi\right)^{-1} \Phi^{\dagger} \geq 0 .
$$

According to the property of positive matrix, we can multiply Eq. (10) by $\Phi^{\dagger}$ from the left side and by $\Phi$ from right side, therefore immediately get the first inequality in Eq. (8). The second inequality of Eq. (8) must be satisfied since the success probabilities $p_{i} \geq 0(i=1, \cdots, n)$ must be non-negative.

ii) Let $\boldsymbol{p}_{1}$ and $\boldsymbol{p}_{2}$ denote two arbitrary points in $\mathcal{S}$, and $\Gamma_{1}, \Gamma_{2}$ denote the diagonal matrices with the components of $\boldsymbol{p}_{1}$ and $\boldsymbol{p}_{2}$ as their diagonal elements, respectively, then $X-\Gamma_{1} \geq 0, \Gamma_{1} \geq 0, X-\Gamma_{2} \geq 0, \Gamma_{2} \geq 0$. Let $\epsilon$ be an arbitrary real number between 0 and 1 and

$$
\boldsymbol{p}_{\epsilon}=\epsilon \boldsymbol{p}_{1}+(1-\epsilon) \boldsymbol{p}_{2}
$$




$$
\Gamma_{\epsilon}=\epsilon \Gamma_{1}+(1-\epsilon) \Gamma_{2}
$$

Since the sum of two positive matrices is still a positive matrix, we have

$$
\begin{gathered}
X-\Gamma_{\epsilon}=X-\left(\epsilon \Gamma_{1}+(1-\epsilon) \Gamma_{2}\right)=\epsilon\left(X-\Gamma_{1}\right)+(1-\epsilon)\left(X-\Gamma_{2}\right) \geq 0, \\
\Gamma_{\epsilon}=\epsilon \Gamma_{1}+(1-\epsilon) \Gamma_{2} \geq 0,
\end{gathered}
$$

which means that $\boldsymbol{p}_{\epsilon} \in \mathcal{S}$. Thus $\mathcal{S}$ is a convex set.

Before deriving more properties of the optimum solution for unambiguous discrimination, we introduce some notations and nomenclatures that will be used later on here.

Let $\sigma_{1}, \cdots, \sigma_{n}$ denote the eigenvalues of $X-\Gamma$ in decreasing order such that $\sigma_{1} \geq \cdots \geq \sigma_{n}$, then the minimum eigenvalue $\sigma_{n}$ must satisfy $\sigma_{n} \geq 0$ due to the positivity of $X-\Gamma$ according to Theorem 1 Since $X-\Gamma$ depends on the parameters $\left\{p_{1}, \cdots, p_{n}\right\}$, which is denoted by a real vector $\boldsymbol{p}$ for short, we have $\sigma_{i}=\sigma_{i}(\boldsymbol{p}),(i=1, \cdots, n)$.

For the convenience of description, the following notations and nomenclatures will be used throughout this article.

- a point: a vector $\boldsymbol{p}$ in $\mathbb{R}^{n}$;

- the feasible set: the set $\mathcal{S}$ defined in Theorem 1 .

- the critical feasible region (denoted by $\mathcal{R}_{C F}$ ): the set of points $\boldsymbol{p}$ in $\mathcal{S}$ satisfying $\sigma_{n}(\boldsymbol{p})=0$ and $\Gamma \geq 0$;

- the boundary of the critical feasible region (denoted by $\mathcal{B}_{R}$ ): the set of points $\boldsymbol{p}$ in the critical feasible region $\mathcal{R}_{C F}$ with at least one zero component, i.e., $p_{i}=0$ for at least one $i \in\{1, \cdots, n\}$;

- the interior part of the critical feasible region (denoted by $\Omega_{R}$ ): the set of points $\boldsymbol{p}$ in the critical feasible region $\mathcal{R}_{C F}$ with $p_{i}>0$ for all $i \in\{1, \cdots, n\}$, i.e. $\Omega_{R}=\mathcal{R}_{C F} \backslash \mathcal{B}_{R}$ where "\" denotes the set exclusion operator;

- an interior point: a point in the interior part $\Omega_{R}$ of the critical feasible region (but not off the critical feasible region);

- a boundary point: a point on the boundary $\mathcal{B}_{R}$ of the critical feasible region;

- a singular point: a point in the critical feasible region where $\nabla \sigma_{n}(\boldsymbol{p})$ does not exist (i.e. $\sigma_{n}(\boldsymbol{p})$ is degenerate) or $\nabla \sigma_{n}(\boldsymbol{p})=\mathbf{0}$. Here $\nabla$ denotes the gradient operator.

It is clear that $\mathcal{B}_{R} \cup \Omega_{R}=\mathcal{R}_{C F}$, and $\mathcal{R}_{C F} \subset \mathcal{S}$.

We give an example of three pure states below to explain the nomenclatures defined above intuitively by graphics. Suppose the states to be discriminated are

$$
\left|\psi_{1}\right\rangle=(1,0,0)^{T},\left|\psi_{2}\right\rangle=\frac{1}{\sqrt{5}}(1,2,0)^{T},\left|\psi_{3}\right\rangle=\frac{2}{\sqrt{17}}\left(1,1, \frac{3}{2}\right)^{T} .
$$

Fig. 1 shows the feasible set $\mathcal{S}$, the critical feasible region $\mathcal{R}_{C F}$, the boundary $\mathcal{B}_{R}$ of the critical feasible region and the singular point $\boldsymbol{p}_{S}$ for these three states. But it should be noted that the singular point does not always exist for every set of states. A necessary condition for the existence of a singular point in the case of three states is given in Case 3 of Sec. VIB. In addition, Fig. 1 explicitly shows that the feasible set is convex, which agrees with Theorem 1.

Now we give two lemmas that will be used in the proofs of the next several theorems.

Lemma 1. If there exists a point $\boldsymbol{p}_{0}$ in the critical feasible region $\mathcal{R}_{C F}$ satisfying that $\boldsymbol{\gamma} \cdot \boldsymbol{p} \leq \boldsymbol{\gamma} \cdot \boldsymbol{p}_{0}$ for any point $\boldsymbol{p}$ in a sufficiently small neighborhood $\Delta$ of $\boldsymbol{p}_{0}, \Delta \subset \mathcal{S}$, then $\boldsymbol{\gamma} \cdot \boldsymbol{p} \leq \boldsymbol{\gamma} \cdot \boldsymbol{p}_{0}$ holds for any point $\boldsymbol{p} \in \mathcal{S}$.

Proof. By contradiction. Suppose there exists such a point $\boldsymbol{p}_{1} \in \mathcal{S}$ that $\boldsymbol{\gamma} \cdot \boldsymbol{p}_{1}>\boldsymbol{\gamma} \cdot \boldsymbol{p}_{0}$, let $\epsilon$ be a sufficiently small positive real number satisfying $0<\epsilon<1$, and let

$$
\boldsymbol{p}_{\epsilon}=\epsilon \boldsymbol{p}_{1}+(1-\epsilon) \boldsymbol{p}_{0},
$$

then $\boldsymbol{p}_{\epsilon} \in \mathcal{S}$ because of the convexity of $\mathcal{S}$ proved in Theorem 1, and $\boldsymbol{p}_{\epsilon} \in \Delta$ since $\epsilon$ is sufficiently small. However,

$$
\boldsymbol{\gamma} \cdot \boldsymbol{p}_{\epsilon}=\boldsymbol{\gamma} \cdot\left(\epsilon \boldsymbol{p}_{1}+(1-\epsilon) \boldsymbol{p}_{0}\right)=\epsilon \boldsymbol{\gamma} \cdot \boldsymbol{p}_{1}+(1-\epsilon) \gamma \cdot \boldsymbol{p}_{0}>\gamma \cdot \boldsymbol{p}_{0}
$$

which contradicts the assumption of this lemma. Therefore, $\boldsymbol{\gamma} \cdot \boldsymbol{p} \leq \boldsymbol{\gamma} \cdot \boldsymbol{p}_{0}$ holds for any point $\boldsymbol{p}$ in $\mathcal{S}$ 


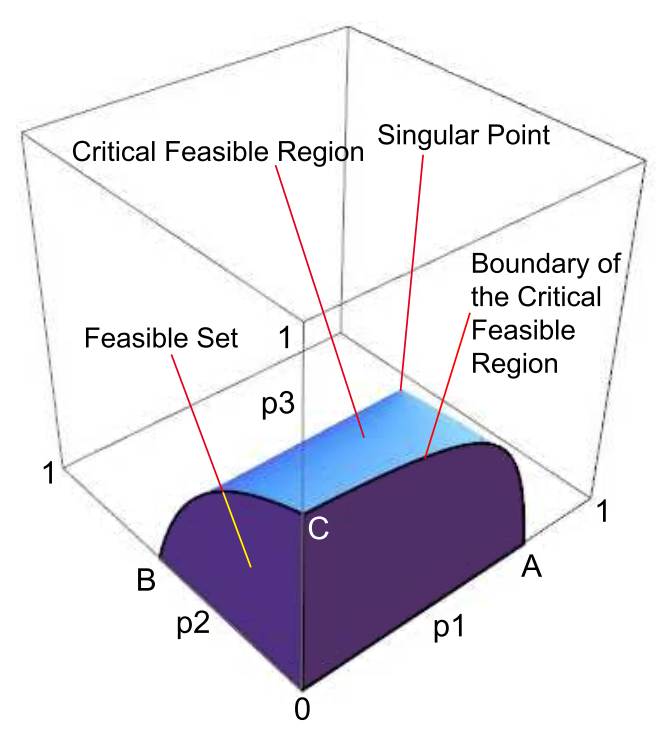

Figure 1: (Color online)Feasible Set, critical feasible region, boundary and singular point. The whole shaded volume (including its surface) is the feasible set $\mathcal{S}$, the upper curved surface of the feasible set is the critical feasible region $\mathcal{R}_{C F}$, and the black curved boundary line $\overparen{B C} \cup \overparen{A C} \cup \overparen{A B}(\overparen{A B}$ is hidden and invisible in the figure $)$ is the boundary $\mathcal{B}_{R}$ of the critical feasible region. The singular point is the point at which the curved surface is not smooth.

Intuitively, Lemma 1 tells us that if a linear function acting on a convex set achieves a local maximal value at some point of the set, then that local maximal point must be the global maximum point that the function can reach over the whole set.

Lemma 2. Suppose $\boldsymbol{a}$ and $\boldsymbol{b}$ are two non-zero real vectors in the $\mathbb{R}^{n}$ space. $(\boldsymbol{a} \cdot \boldsymbol{x})(\boldsymbol{b} \cdot \boldsymbol{x}) \leq 0$ for any vector $\boldsymbol{x}$, if and only if $\boldsymbol{a}$ and $\boldsymbol{b}$ are anti-parallel, i.e., $\boldsymbol{a}=-\lambda \boldsymbol{b}$ where $\lambda$ is positive factor.

Proof. Necessity: By contradiction. Let $\boldsymbol{x}=\boldsymbol{a}_{0}+\boldsymbol{b}_{0}$, where $\boldsymbol{a}_{0}=\frac{\boldsymbol{a}}{|\boldsymbol{a}|}$ and $\boldsymbol{b}_{0}=\frac{\boldsymbol{b}}{|\boldsymbol{b}|}$. Let $\theta$ denote the angular between $\boldsymbol{a}$ and $\boldsymbol{b}$, i.e., $\boldsymbol{a} \cdot \boldsymbol{b}=|\boldsymbol{a}||\boldsymbol{b}| \cos \theta$. If $\boldsymbol{a}$ and $\boldsymbol{b}$ are not anti-parallel, then $0 \leq \theta<\pi$ by the well known Cauchy-Schwartz inequality [58] and $\boldsymbol{x} \neq \mathbf{0}$. Then we have $(\boldsymbol{a} \cdot \boldsymbol{x})(\boldsymbol{b} \cdot \boldsymbol{x})=|\boldsymbol{a} \| \boldsymbol{b}|(1+\cos \theta)^{2}>0$, contradictory to the assumption.

Sufficiency: If $\boldsymbol{a}$ and $\boldsymbol{b}$ are anti-parallel, i.e., $\boldsymbol{a}=-\lambda \boldsymbol{b}(\lambda>0)$, then for any non-zero $\boldsymbol{x},(\boldsymbol{a} \cdot \boldsymbol{x})(\boldsymbol{b} \cdot \boldsymbol{x})=-\lambda(\boldsymbol{b} \cdot \boldsymbol{x})^{2} \leq$ 0 .

Now we are going to show some important properties of the optimum point, from which we will establish two sets of new equations that the optimum solution has to satisfy later in different situations.

Theorem 2(Minimum Eigenvalue). i) The optimum point $\boldsymbol{p}_{\text {opt }}$ must be a point in the critical feasible region $\mathcal{R}_{C F}$, i.e.,

$$
\sigma_{n}\left(\boldsymbol{p}_{\text {opt }}\right)=0
$$

ii) If the optimum point $\boldsymbol{p}_{\text {opt }}$ is a non-singular point in the interior part $\Omega_{R}$ of the critical feasible region, $\boldsymbol{p}_{\text {opt }} \in \Omega_{R}$, then

$$
\left.\nabla \sigma_{n}(\boldsymbol{p})\right|_{\boldsymbol{p}_{\text {opt }}}=-\gamma
$$

iii) Conversely, if there exists a point $\boldsymbol{p}_{0}$ satisfying $\left.\nabla \sigma_{n}(\boldsymbol{p})\right|_{\boldsymbol{p}_{0}}=-\boldsymbol{\gamma}$ in the critical feasible region $\mathcal{R}_{C F}, \boldsymbol{p}_{0}$ must be the global optimum point.

Proof. i) Since $\sigma_{n}$ is the minimum eigenvalue of the matrix $X-\Gamma$ and $X-\Gamma$ is positive as proved in Theorem 1 , we have $\sigma_{n} \geq 0$. Thus, in order to prove Eq. (18), we only need to show that $\sigma_{n}$ cannot be greater than zero at the optimum point. We prove it by contradiction.

If $\sigma_{n}\left(\boldsymbol{p}_{\text {opt }}\right)>0$, we let $\boldsymbol{p}_{\text {opt }}$ be changed a little by $\delta \boldsymbol{p}$, then we can always find such $\delta \boldsymbol{p}$ that $\boldsymbol{\gamma} \cdot \delta \boldsymbol{p}>0$ and at the same time $\sigma_{n}\left(\boldsymbol{p}_{\text {opt }}+\delta \boldsymbol{p}\right) \geq 0, \Gamma+\delta \Gamma \geq 0$, where $\delta \Gamma$ is a diagonal matrix with $\delta p_{1}, \cdots, \delta p_{n}$ as its diagonal elements (such $\delta \boldsymbol{p}$ always exists as we can construct it simply by taking $\delta p_{i}>0$ and $\delta p_{i}$ sufficiently small, for all $i=1, \cdots, n$ ). This implies that the point $\boldsymbol{p}_{\text {opt }}+\delta \boldsymbol{p}$ in the feasible set $\mathcal{S}$ satisfies $\boldsymbol{\gamma} \cdot\left(\boldsymbol{p}_{\text {opt }}+\delta \boldsymbol{p}\right)>\boldsymbol{\gamma} \cdot \boldsymbol{p}_{\text {opt }}$, which contradicts the assumption that $\gamma \cdot \boldsymbol{p}$ reaches the maximum at $\boldsymbol{p}_{\text {opt }}$, so Eq. (18) holds.

It seems that the above proof may be applied to other eigenvalues of $X-\Gamma$ in a similar way, but if any eigenvalue other than the minimal one is equal to zero, then the minimal eigenvalue would be negative and $X-\Gamma$ would not keep positive, violating Eq. (8). 
ii) Since $\boldsymbol{p}_{\text {opt }}$ is an interior optimum point in the critical feasible region $\mathcal{R}_{C F}$, if we change $\boldsymbol{p}_{\text {opt }}$ a little by any $\delta \boldsymbol{p}$ such that $\sigma_{n}\left(\boldsymbol{p}_{\text {opt }}+\delta \boldsymbol{p}\right) \geq 0$, it must be that $\boldsymbol{\gamma} \cdot \delta \boldsymbol{p} \leq 0$, otherwise $\boldsymbol{\gamma} \cdot \boldsymbol{p}$ is not maximal at $\boldsymbol{p}_{\text {opt }}$. Considering $\sigma_{n}\left(\boldsymbol{p}_{\text {opt }}\right)=0$, the inequality $\sigma_{n}\left(\boldsymbol{p}_{\text {opt }}+\delta \boldsymbol{p}\right) \geq 0$ can be converted to

$$
\left.\delta \sigma_{n}(\boldsymbol{p})\right|_{\boldsymbol{p}_{\text {opt }}} \geq 0 \text {. }
$$

Since $\boldsymbol{p}_{\text {opt }}$ is not a singular point, $\left.\nabla \sigma_{n}(\boldsymbol{p})\right|_{\boldsymbol{p}_{\text {opt }}}$ exists, so

$$
\delta \sigma_{n}(\boldsymbol{p})=\sigma_{n}\left(\boldsymbol{p}_{\text {opt }}+\delta \boldsymbol{p}\right)-\sigma_{n}\left(\boldsymbol{p}_{\text {opt }}\right)=\left.\nabla \sigma_{n}(\boldsymbol{p})\right|_{\boldsymbol{p}_{\text {opt }}} \cdot \delta \boldsymbol{p} .
$$

Substituting Eq. (21) into (20), we have

$$
\left.\nabla \sigma_{n}(\boldsymbol{p})\right|_{\boldsymbol{p}_{\text {opt }}} \cdot \delta \boldsymbol{p} \geq 0 .
$$

Comparing Eq. (22) with $\gamma \cdot \delta \boldsymbol{p} \leq 0$, it follows that $\gamma$ and $\left.\nabla \sigma_{n}(\boldsymbol{p})\right|_{\boldsymbol{p}_{\text {opt }}}$ must be anti-parallel according to Lemma 2 , so

$$
\left.\nabla \sigma_{n}(\boldsymbol{p})\right|_{\boldsymbol{p}_{\text {opt }}}=-\alpha \gamma, \alpha>0
$$

We will prove that $\alpha=1$ in the proof of Theorem 3, thus Eq. (19) holds.

iii) If there exists a point $\boldsymbol{p}_{0}$ where $\left.\nabla \sigma_{n}(\boldsymbol{p})\right|_{\boldsymbol{p}_{0}}=-\boldsymbol{\gamma}$ in the critical feasible region $\mathcal{R}_{C F}$, any small $\delta \boldsymbol{p}$ such that $\sigma_{n}\left(\boldsymbol{p}_{0}+\delta \boldsymbol{p}\right)=\delta \sigma_{n}(\boldsymbol{p})=\left.\nabla \sigma_{n}(\boldsymbol{p})\right|_{\boldsymbol{p}_{0}} \cdot \delta \boldsymbol{p} \geq 0$ will lead to $\boldsymbol{\gamma} \cdot \delta \boldsymbol{p}=-\left.\nabla \sigma_{n}(\boldsymbol{p})\right|_{\boldsymbol{p}_{0}} \cdot \delta \boldsymbol{p} \leq 0$, so $\gamma \cdot \boldsymbol{p}$ reaches maximal at $\boldsymbol{p}_{0}$ in a small neighborhood of $\boldsymbol{p}_{0}$, and according to Lemma 1, $\boldsymbol{p}_{0}$ must be the global optimum point where $\gamma \cdot \boldsymbol{p}$ reaches the global maximum.

It should be pointed out that a result equivalent to our result i) in Theorem 2 was obtained in Ref. [31], where the maximum eigenvalue of $\widetilde{\Phi} \Gamma \widetilde{\Phi}^{\dagger}$ is proved to be exactly 1 when the unambiguous state discrimination scheme is optimum. Besides, Eq. (18) implies that

$$
\operatorname{det}(X-\Gamma)=0
$$

at the optimum point, and a physical interpretation of this fact was given in [34].

To prove the next theorem, we first give another lemma [59].

Lemma 3. If $\tau_{1}, \cdots, \tau_{n}$ are the eigenvalues of an $n \times n$ matrix $A$, then $\sum_{1 \leq i_{1}<\cdots<i_{k} \leq n} \tau_{i_{1}} \cdots \tau_{i_{k}}=$ $\sum($ all $k \times k$ principal minors of $A)$.

Based on Theorem 2, we now present the following theorem which can be conveniently used to obtain the maximum average success probability and the optimum measurement strategy when the optimum point is not singular and in the interior part $\Omega_{R}$ of the critical feasible region.

Theorem 3(Interior Non-Singular Solution). Let $M_{k}(\boldsymbol{p})$ denote the principle minor of order $n-1$ associated with the $k$ th diagonal element of $X-\Gamma$, then a non-singular point $\boldsymbol{p}_{0}$ in the interior part $\Omega_{R}$ of the critical feasible region is the optimum point if and only if $\boldsymbol{p}_{0}$ is a solution of the following set of equations

$$
\left\{\begin{array}{c}
M_{1}(\boldsymbol{p})=\gamma_{1} \lambda \\
\vdots \\
M_{n}(\boldsymbol{p})=\gamma_{n} \lambda \\
\operatorname{det}(X-\Gamma)=0
\end{array}\right.
$$

for some positive number $\lambda>0$, and satisfies the positivity condition (8) at the same time.

Proof. First, we prove the "only if" part of the theorem. Let's consider the variation of $\operatorname{det}(X-\Gamma)$. Since $\operatorname{det}(X-\Gamma)=\sigma_{1} \cdots \sigma_{n}$, we have

$$
\delta \operatorname{det}(X-\Gamma)=\left(\delta \sigma_{1}\right) \sigma_{2} \cdots \sigma_{n}+\sigma_{1}\left(\delta \sigma_{2}\right) \sigma_{3} \cdots \sigma_{n}+\cdots+\sigma_{1} \sigma_{2} \cdots \sigma_{n-1} \delta \sigma_{n}
$$

If $\boldsymbol{p}_{0}$ is the optimum point that achieves the maximum average probability, then $\sigma_{n}\left(\boldsymbol{p}_{0}\right)=0$ according to Theorem 2. Eq. (26) can be simplified to

$$
\left.\delta \operatorname{det}(X-\Gamma)\right|_{\boldsymbol{p}_{0}}=\left.\left(\sigma_{1} \sigma_{2} \cdots \sigma_{n-1} \delta \sigma_{n}\right)\right|_{\boldsymbol{p}_{0}} .
$$

Since $\boldsymbol{p}_{0}$ is not a singular point, $\left.\nabla \sigma_{n}(\boldsymbol{p})\right|_{\boldsymbol{p}_{0}}$ exists and $\left.\delta \sigma_{n}(\boldsymbol{p})\right|_{\boldsymbol{p}_{0}}=\left.\nabla \sigma_{n}(\boldsymbol{p})\right|_{\boldsymbol{p}_{0}} \cdot \delta \boldsymbol{p}$. From $\delta$ det $(X-\Gamma)=$ $\nabla \operatorname{det}(X-\Gamma) \cdot \delta \boldsymbol{p}$ and Eq. (27), we have

$$
\left.\nabla \operatorname{det}(X-\Gamma)\right|_{\boldsymbol{p}_{0}}=\left.\sigma_{1} \sigma_{2} \cdots \sigma_{n-1} \nabla \sigma_{n}(\boldsymbol{p})\right|_{\boldsymbol{p}_{0}} .
$$


Again using $\sigma_{n}\left(\boldsymbol{p}_{0}\right)=0$, we can get

$$
\left.\nabla \operatorname{det}(X-\Gamma)\right|_{\boldsymbol{p}_{0}}=\left.\left(\sigma_{1} \cdots \sigma_{n-1}+\sigma_{1} \cdots \sigma_{n-2} \sigma_{n}+\cdots+\sigma_{2} \cdots \sigma_{n}\right) \nabla \sigma_{n}(\boldsymbol{p})\right|_{\boldsymbol{p}_{0}} .
$$

According to Lemma 3, we have

$$
\left.\nabla \operatorname{det}(X-\Gamma)\right|_{\boldsymbol{p}_{0}}=\left.\left(M_{1}\left(\boldsymbol{p}_{0}\right)+\cdots+M_{n}\left(\boldsymbol{p}_{0}\right)\right) \nabla \sigma_{n}(\boldsymbol{p})\right|_{\boldsymbol{p}_{0}}
$$

On the other hand,

$$
\nabla \operatorname{det}(X-\Gamma)=\left(e_{1} \frac{\partial}{\partial p_{1}}+\cdots+e_{n} \frac{\partial}{\partial p_{n}}\right) \operatorname{det}(X-\Gamma),
$$

where $\boldsymbol{e}_{i}$ is the orthonormal basis vector in the space $\mathbb{R}^{n}$ associated with the coordinate $p_{i}$. By performing Laplace expansion on the determinant of $X-\Gamma$ along the $k$ th row (or column), we can see that

$$
\frac{\partial}{\partial p_{k}} \operatorname{det}(X-\Gamma)=-M_{k}(\boldsymbol{p})
$$

SO

$$
\nabla \operatorname{det}(X-\Gamma)=-\left(M_{1}(\boldsymbol{p}) \boldsymbol{e}_{1}+\cdots+M_{n}(\boldsymbol{p}) \boldsymbol{e}_{n}\right)
$$

Comparing Eq. (30) and (33) and substituting Eq. (23) into (30), we have

$$
-\left(M_{1}\left(\boldsymbol{p}_{0}\right) \boldsymbol{e}_{1}+\cdots+M_{n}\left(\boldsymbol{p}_{0}\right) \boldsymbol{e}_{n}\right)=-\left(M_{1}\left(\boldsymbol{p}_{0}\right)+\cdots+M_{n}\left(\boldsymbol{p}_{0}\right)\right) \alpha \boldsymbol{\gamma}
$$

which results in

$$
M_{k}\left(\boldsymbol{p}_{0}\right)=\alpha \gamma_{k}\left(M_{1}\left(\boldsymbol{p}_{0}\right)+\cdots+M_{n}\left(\boldsymbol{p}_{0}\right)\right), k=1, \cdots, n
$$

Considering that $X-\Gamma \geq 0$, we have $M_{k}\left(\boldsymbol{p}_{0}\right) \geq 0$ for all $k=1, \cdots, n$. Since the theorem assumes that the optimum point is not singular, the zero eigenvalue is not degenerate and $\left.\nabla \sigma_{n}(\boldsymbol{p})\right|_{\boldsymbol{p}_{0}} \neq \mathbf{0}$, then according to Eq. (28), we have

$$
\left.\nabla \operatorname{det}(X-\Gamma)\right|_{p_{0}} \neq \mathbf{0}
$$

So according to Eq. (33) we can see that the $M_{k}\left(\boldsymbol{p}_{0}\right)$ 's are not all zeros and

$$
M_{1}\left(\boldsymbol{p}_{0}\right)+\cdots+M_{n}\left(\boldsymbol{p}_{0}\right)>0
$$

By summing up Eq. (35) for $i=1, \cdots, n$ and using $\gamma_{1}+\cdots \gamma_{n}=1$ and Eq. (37), we immediately have $\alpha=1$. (This completes the remaining part of the proof for part ii) of Theorem 2])

Thus, Eq. (35) can be simplified to

$$
M_{k}\left(\boldsymbol{p}_{0}\right)=\gamma_{k}\left(M_{1}\left(\boldsymbol{p}_{0}\right)+\cdots+M_{n}\left(\boldsymbol{p}_{0}\right)\right), k=1, \cdots, n
$$

which immediately leads to Eq. (25). Considering Eq. (37), there must be $\lambda>0$ according to Eq. (25).

This concludes the proof of the "only if" part of the theorem.

Next, we prove the "if" part of the theorem. Considering $X-\Gamma \geq 0, \operatorname{det}(X-\Gamma)=0$ in Eq. (25) and that $\sigma_{n}\left(\boldsymbol{p}_{0}\right)$ is the minimal eigenvalue of $X-\Gamma$, it follows straightforwardly that

$$
\sigma_{n}\left(\boldsymbol{p}_{0}\right)=0
$$

And since $\lambda>0, M_{k}\left(\boldsymbol{p}_{0}\right)>0, k=1, \cdots, n$, thus the rank of $X-\Gamma$ is $n-1$ and $\nabla \operatorname{det}(X-\Gamma) \mid \boldsymbol{p}_{0} \neq \mathbf{0}$, which imply that $\boldsymbol{p}_{0}$ is not a singular point. It can be seen that Eq. (30) still holds here, so substituting (25) into Eqs. (30) and (33), one can have

$$
-\left(\gamma_{1} \lambda \boldsymbol{e}_{1}+\cdots+\gamma_{n} \lambda \boldsymbol{e}_{n}\right)=\left.\lambda \nabla \sigma_{n}(\boldsymbol{p})\right|_{\boldsymbol{p}_{0}}
$$

where $\gamma_{1}+\cdots \gamma_{n}=1$ has been used. Therefore we get $\left.\nabla \sigma_{n}(\boldsymbol{p})\right|_{\boldsymbol{p}_{0}}=-\boldsymbol{\gamma}$, and together with (39) it implies that $\boldsymbol{p}_{0}$ is the (global) optimum point according to part iii) of Theorem 2. This concludes the proof of the "if" part of the theorem. 
Theorem 2 and Theorem 3 describe the properties of the optimum point when it is a non-singular point in the interior part $\Omega_{R}$ of the critical feasible region, and Theorem 3 also gives a way to find such an optimum point. When one obtains a solution from Eq. (25) for some $\lambda>0$, he or she has to verify whether it satisfies Eq. (8). If it does, this solution is the optimum solution. However, if (25) has no solution satisfying $\lambda>0$ and (8), then the optimum point is either a point on the boundary $\mathcal{B}_{R}$ or a singular point (if it exists) of the critical feasible region $\mathcal{R}_{C F}$. The next theorem is to characterize the properties of the optimum point when it is on the boundary $\mathcal{B}_{R}$ and give a method to work out the optimum point in that situation.

For the simplicity of later description, we further define some new notations here. We denote the part of the critical feasible region $\mathcal{R}_{C F}$ where $p_{i_{1}}=0, \cdots, p_{i_{k}}=0$ as $\mathcal{B}_{R}\left(i_{1}, \cdots, i_{k}\right)$, and any $\mathcal{B}_{R}\left(i_{1}, \cdots, i_{k}\right)$ will be called a $(n-k-1)$-dimensional boundary of the critical feasible region (since the critical feasible region itself is of dimension $n-1$ in the $\mathbb{R}^{n}$ space). We again take the three state (15) as an example. In Fig. 1 the black boundary line excluding the points $\mathrm{A}, \mathrm{B}$ and $\mathrm{C}$ is the 1-dimensional boundary of the critical feasible region, and the points $\mathrm{A}, \mathrm{B}$ and $\mathrm{C}$ are the 0-dimensional boundaries of the critical feasible region.

Theorem 4(Boundary Solution). A point $\boldsymbol{p}_{0}$ on a $\left(n-k_{0}-1\right)$-dimensional boundary $\mathcal{B}_{R}\left(i_{1}, \cdots, i_{k_{0}}\right)$ but not on any lower dimensional boundary, i.e., $p_{i_{1}}=0, \cdots, p_{i_{k_{0}}}=0$ and $p_{j}>0, \forall j \neq i_{1}, \cdots, i_{k_{0}}$, is the optimum point if and only if it is a solution of

$$
\left\{\begin{array}{l}
\left.M_{i}(\boldsymbol{p})\right|_{p_{i_{1}}=0, \cdots, p_{i_{k_{0}}}=0}=\gamma_{i} \lambda \quad \forall i \in\{1, \cdots, n\} \backslash\left\{i_{1}, \cdots, i_{k_{0}}\right\} \\
\left.\operatorname{det}(X-\Gamma)\right|_{p_{i_{1}}=0, \cdots, p_{i_{0}}}=0
\end{array},\right.
$$

satisfying $\lambda>0$, the positivity constraints (8) and

$$
\left\{\begin{array}{l}
\left.M_{i_{1}}(\boldsymbol{p})\right|_{p_{i_{1}}=0, \cdots, p_{i_{k_{0}}}}=0 \\
\quad \vdots \\
\left.M_{i_{k_{0}}}(\boldsymbol{p})\right|_{p_{i_{1}}=0, \cdots, p_{i_{k_{0}}}=0} \geq \lambda \gamma_{i_{1}} \\
\quad .
\end{array}\right.
$$

Proof. First, we prove the "only if" part of the theorem. If $\boldsymbol{p}_{0}$ is an optimum point on the $\left(n-k_{0}-1\right)$-dimensional boundary $\mathcal{B}_{R}\left(i_{1}, \cdots, i_{k_{0}}\right)$ but not on any lower dimensional boundary, let $\boldsymbol{p}_{0}$ be changed to $\boldsymbol{p}_{0}+\delta \boldsymbol{p}$ on $\mathcal{B}_{R}\left(i_{1}, \cdots, i_{k_{0}}\right)$ by any small $\delta \boldsymbol{p}$ satisfying $\delta p_{i_{1}}=0, \cdots, \delta p_{i_{k_{0}}}=0$, then there must be

$$
\left.\delta \operatorname{det}(X-\Gamma)\right|_{\boldsymbol{p}_{0}}=\left.\nabla \operatorname{det}(X-\Gamma)\right|_{\boldsymbol{p}_{0}} \cdot \delta \boldsymbol{p}=-\sum_{i \neq i_{1}, \cdots, i_{k_{0}}} M_{i}\left(\boldsymbol{p}_{0}\right) \delta p_{i}=0,
$$

where we have used Eq. (33), and

$$
\boldsymbol{\gamma} \cdot \delta \boldsymbol{p}=\sum_{i \neq i_{1}, \cdots, i_{k_{0}}} \gamma_{i} \delta p_{i} \leq 0
$$

So

$$
\left.\left.\left(M_{1}\left(\boldsymbol{p}_{0}\right), \cdots, M_{i}\left(\boldsymbol{p}_{0}\right), \cdots, M_{n}\left(\boldsymbol{p}_{0}\right)\right)\right|_{i \neq i_{1}, \cdots, i_{k_{0}}} \propto\left(\gamma_{1}, \cdots, \gamma_{i}, \cdots, \gamma_{n}\right)\right|_{i \neq i_{1}, \cdots, i_{k_{0}}}
$$

according to Lemma 2, therefore, Eq. (41) holds.

On the other hand, let $\boldsymbol{p}_{0}$ be changed to $\boldsymbol{p}_{0}+\delta \boldsymbol{p}^{\prime}$ in the critical feasible region $\mathcal{R}_{C F}$ by another arbitrary small $\delta \boldsymbol{p}^{\prime}$ satisfying that $\delta p_{i_{1}}^{\prime} \geq 0, \cdots, \delta p_{i_{k_{0}}}^{\prime} \geq 0$, then there must be

$$
\begin{aligned}
\left.\delta \operatorname{det}(X-\Gamma)\right|_{\boldsymbol{p}_{0}} & =-\sum_{i=1}^{n} M_{i}\left(\boldsymbol{p}_{0}\right) \delta p_{i}^{\prime} \\
& =-\sum_{i=i_{1}, \cdots, i_{k_{0}}} M_{i}\left(\boldsymbol{p}_{0}\right) \delta p_{i}^{\prime}-\sum_{i \neq i_{1}, \cdots, i_{k_{0}}} M_{i}\left(\boldsymbol{p}_{0}\right) \delta p_{i}^{\prime} \\
& =-\sum_{i=i_{1}, \cdots, i_{k_{0}}} M_{i}\left(\boldsymbol{p}_{0}\right) \delta p_{i}^{\prime}-\sum_{i \neq i_{1}, \cdots, i_{k_{0}}} \lambda \gamma_{i} \delta p_{i}^{\prime}=0
\end{aligned}
$$

where we have used Eq. (41), and

$$
\boldsymbol{\gamma} \cdot \delta \boldsymbol{p}^{\prime}=\sum_{i=1}^{n} \gamma_{i} \delta p_{i}^{\prime}=\sum_{i=i_{1}, \cdots, i_{k_{0}}} \gamma_{i} \delta p_{i}^{\prime}+\sum_{i \neq i_{1}, \cdots, i_{k_{0}}} \gamma_{i} \delta p_{i}^{\prime} \leq 0 .
$$


Substituting Eq. (46) into (47), we have

$$
\boldsymbol{\gamma} \cdot \delta \boldsymbol{p}^{\prime}=\sum_{i=1}^{n} \gamma_{i} \delta p_{i}^{\prime}=\sum_{i=i_{1}, \cdots, i_{k_{0}}}\left(\gamma_{i}-\frac{M_{i}\left(\boldsymbol{p}_{0}\right)}{\lambda}\right) \delta p_{i}^{\prime} \leq 0 .
$$

Considering $\delta p_{i_{1}}^{\prime} \geq 0, \cdots, \delta p_{i_{k_{0}}}^{\prime} \geq 0$ and $\lambda>0$, it can been seen that Eq. (42) holds according to Eq. (48). This concludes the proof of the "only if" part of the theorem.

The "if" part of the theorem can be directly proved by reversing the above reasoning and using Lemma 1, so we are not going to show the details here.

Since the feasible set $\mathcal{S}$ is a closed convex set confined in a finite region of $\mathbb{R}^{n}$, the optimum point where the average success probability $\boldsymbol{\gamma} \cdot \boldsymbol{p}$ reaches the maximum always exists. Thus if Eq. (41) for any $\left(n-k_{0}-1\right)$-dimensional boundary $\left(1 \leq k_{0} \leq n-1\right)$ does not have a solution satisfying $\lambda>0$, the positivity constraints (8) and Eq. (42) while the optimum point is also not an interior non-singular point in the critical feasible region $\mathcal{R}_{C F}$, the optimum point can only be a singular point then. We know that a singular point is a point in the critical feasible region where $\sigma_{n}(\boldsymbol{p})=0$ is degenerate or $\nabla \sigma_{n}(\boldsymbol{p})=\mathbf{0}$, so when the optimum point is singular, $\left.\nabla \operatorname{det}(X-\Gamma)\right|_{\boldsymbol{p}_{0}}=\mathbf{0}$ according to Eq. (28), which, together with (33), implies that all $M_{k}(\boldsymbol{p})=0$ for all $k=1, \cdots, n$. Thus a singular point can be obtained as a solution of (25) with $\lambda=0$ and the positivity conditions (8).

Remark 1. It should be pointed out that given a set of linearly independent states, if there exists a singular point in the critical feasible region $\mathcal{R}_{C F}$, then that singular point could be the optimum point for a range of different $\gamma^{\prime}$, since the normal vector of the critical feasible region $\mathcal{R}_{C F}$ changes discontinuously in the neighborhood of a singular point.

In this subsection we have mainly studied the properties of the optimum point and obtained the equations that the optimum point must satisfy in different situations. We summarize our method to find the optimum point as follows:

Step I. Try to solve Eq. (25) in Theorem 3and see whether there exists a solution satisfying the positivity constraints $\lambda>0$ and Eq. (8). If such a solution exists, it is exactly the optimum point we try to find.

Step II. If the set of equations (25) does not have a solution that satisfies $\lambda>0$ and Eq. (8), one has to continue to search for the optimum point on the boundary $\mathcal{B}_{R}$ of the critical feasible region using Theorem 4 In detail, one can first solve Eq. (41) on all $(n-2)$-dimensional boundaries and see whether there exists a solution that satisfies $\lambda>0$, the positivity constraints (8) and Eq. (42). If such a solution exists, it is exactly the optimum point; otherwise, one should further search on all $(n-3)$-dimensional boundaries, $(n-4)$-dimensional boundaries, $\ldots$, until such a solution is found or all boundaries of dimension lower than $n-1$ have been searched. If such a solution is found, it is exactly the optimum solution.

Step III. If the optimum point is not found in the above two steps, then it must be a singular point and can be obtained by solving Eq. (25) with $\lambda=0$. If there exists more than one singular point on the critical feasible region $\mathcal{R}_{C F}$, the one that maximizes $\boldsymbol{\gamma} \cdot \boldsymbol{p}$ is the optimum point.

By Theorem 3 and 4 we give two sets of explicit analytical equations for solving the unambiguous discrimination problem in different situations. We can use them to work out analytical solutions or obtain some analytical relations for the problem (see examples in Sec. IV V and VI). However, since Eqs. (25) and (41) are nonlinear and the variables $p_{1}, \cdots, p_{n}$ are tightly coupled in the equations, maybe only numeric solutions can be obtained for these equations in some situations. A lot of sophisticated numerical techniques like Newton's method (including many of its variants), hybrid Krylov methods and so on have been developed to solve such nonlinear equations [60, 61, 62, 63].

It is worth mentioning that other methods such as semidefinite programming [54, 55, 56] have been developed to solve this unambiguous discrimination problem. Those methods are developed from some classical numerical analysis theories and they are mostly suitable for finding numeric solutions, while our method is developed purely by algebra and go in a totally different way, aiming at providing a new tool to treat the problem analytically.

\section{B. Geometrical view and numerical example}

A geometrical method is given in [33] to solve the optimum unambiguous discrimination problem for three pure states, mainly for the situation when the optimum point is an interior non-singular point. In this subsection, we are going to give a similar but more complete geometrical way to illustrate the problem and the results we obtain in the previous subsection, and we calculate a numerical example illustrated with corresponding graphics to explicitly show the geometrical meanings.

Geometrically, $\bar{p}=\boldsymbol{\gamma} \cdot \boldsymbol{p}=\gamma_{1} p_{1}+\cdots+\gamma_{n} p_{n}$ can be perceived as an $(n-1)$-dimensional plane in the $\mathbb{R}^{n}$ space, and the critical feasible region $\sigma_{n}(\boldsymbol{p})=0$ can be perceived as a curved surface in $\mathbb{R}^{n}$. 
Table I: Numerical results of optimum points with different prior probabilities

\begin{tabular}{ccccccc}
\hline Category & Prior Probabilities & $p_{1}$ & $p_{2}$ & $p_{3}$ & $\lambda$ & $\bar{p}_{\text {opt }}$ \\
\hline \hline Interior Point & $\gamma_{1}=0.05, \gamma_{2}=0.35, \gamma_{3}=0.60$ & 0.5029 & 0.3169 & 0.3629 & 0.2326 & 0.3538 \\
\hline Boundary Point & $\gamma_{1}=0.10, \gamma_{2}=0.80, \gamma_{3}=0.10$ & 0.3927 & 0.5300 & 0 & 0.6577 & 0.4632 \\
\hline Singular Point & $\gamma_{1}=0.30, \gamma_{2}=0.35, \gamma_{3}=0.35$ & 0.6667 & 0.4000 & 0.2941 & 0 & 0.4429 \\
\hline
\end{tabular}

It can be shown easily that the vertical distance from the origin of the coordinate system (with $p_{1}, \cdots, p_{n}$ as the coordinates) to the plane $\bar{p}=\boldsymbol{\gamma} \cdot \boldsymbol{p}$ is

$$
\frac{\bar{p}}{\sqrt{\gamma_{1}^{2}+\cdots+\gamma_{n}^{2}}},
$$

so the average success probability $\bar{p}$ characterizes the vertical distance between the origin and the plane $\bar{p}=\gamma \cdot \boldsymbol{p}$ in a geometrical view. Therefore, the problem of optimum unambiguous discrimination of pure states can be translated to the problem of finding an optimum point in the feasible set $\mathcal{S}$ at which the plane with fixed normal vector $\left(\gamma_{1}, \gamma_{2}, \gamma_{3}\right)$ (unnormalized) is most distant from the origin. Obviously the optimum point must lie in the critical feasible region $\mathcal{R}_{C F}$, which is the "surface" of the feasible set $\mathcal{S}$, and this is in accordance with i) of Theorem 2

Now suppose the plane $\bar{p}=\boldsymbol{\gamma} \cdot \boldsymbol{p}$ is moved by parallel shifts, i.e., by changing $\bar{p}$ while the normal vector $\left(\gamma_{1}, \gamma_{2}, \gamma_{3}\right)$ keeps fixed. If the plane can be tangent with the critical feasible region $\mathcal{R}_{C F}$ when $\bar{p}$ is equal to some $\bar{p}_{0}$, the distance from the origin to the plane is then maximized and the tangent point is exactly the optimum point $\boldsymbol{p}_{\text {opt }}$, and $\bar{p}_{0}$ is the maximum average success probability.

When the plane and the critical feasible region $\mathcal{R}_{C F}$ are tangent, their normal vectors at the tangent point should be parallel or anti-parallel. This implies that $\nabla(\boldsymbol{\gamma} \cdot \boldsymbol{p}-\bar{p})=\zeta \nabla \operatorname{det}(X-\Gamma)$, where $\zeta \in \mathbb{R}$ and $\zeta \neq 0$. Using Eq. (33), this equation can be simplified to Eq. (25).

However, if the plane can never be tangent with the critical feasible region when the plane is moved by any parallel shift, it means that a non-singular optimum point does not exist in the interior part of the critical feasible region, then the optimum point is either a boundary point on $\mathcal{B}_{R}$ or a singular point in the critical feasible region. And if the optimum point is on some $(n-k-1)$-dimensional boundary, the plane is then tangent with that $(n-k-1)$ dimensional boundary, resulting in Eq. (41). Conversely, if the plane is tangent with some $(n-k-1)$-dimensional boundary, it does not imply that the tangent point must be the optimum point though, unless Eq. (42) is satisfied, which ensures the average success probability at any other point in the feasible set will be no larger than that at the tangent point, due to the convexity of the feasible set. This gives the geometrical meaning of Theorem 4.

From above, it can be seen that given the states to be discriminated, the category of the optimum point is not determined: it may be a non-singular interior point in the critical feasible region, a point on the boundary of the critical feasible region, or even a singular point, depending on the prior probabilities. Taking the states in (15) as an example, we numerically obtain the optimum points for three different sets of prior probabilities which result in the above three different categories of the optimum points. The results are presented in Table \and the corresponding graphics are Fig. 2, Fig. 3] and Fig. 4 respectively.

\section{ANALYTICAL RELATION BETWEEN THE OPTIMUM SOLUTION AND THE STATES TO BE DISCRIMINATED}

In this section, we shall use the equations established in the previous section to make some efforts on the analytical optimum solution to the problem of unambiguously discriminating $n$ pure states. We shall obtain a formula which is not a complete analytical solution but can characterize a clear relation between the optimum solution and the states to be discriminated, and we shall give an example to show the use of that formula.

\section{A. Formula}

First of all, we give another form of the matrix $X-\Gamma$ in a way similar to Eqs. (2.5) and (2.6) of [34]. Suppose the states $\left|\psi_{k}\right\rangle(k=1, \cdots, n)$ are represented in an orthonormal basis of the Hilbert space $\mathcal{H}$ spanned by $\left\{\left|\psi_{k}\right\rangle\right\}_{k=1}^{n}$, then each $\left|\psi_{k}\right\rangle$ has exactly $n$ components in its representation. If the initial state of the system is $\left|\psi_{k}\right\rangle$, then the state after the inconclusive measurement result can be chosen as

$$
\left|\phi_{k}\right\rangle=\sqrt{\Pi_{0}}\left|\psi_{k}\right\rangle, k=1 \cdots n,
$$




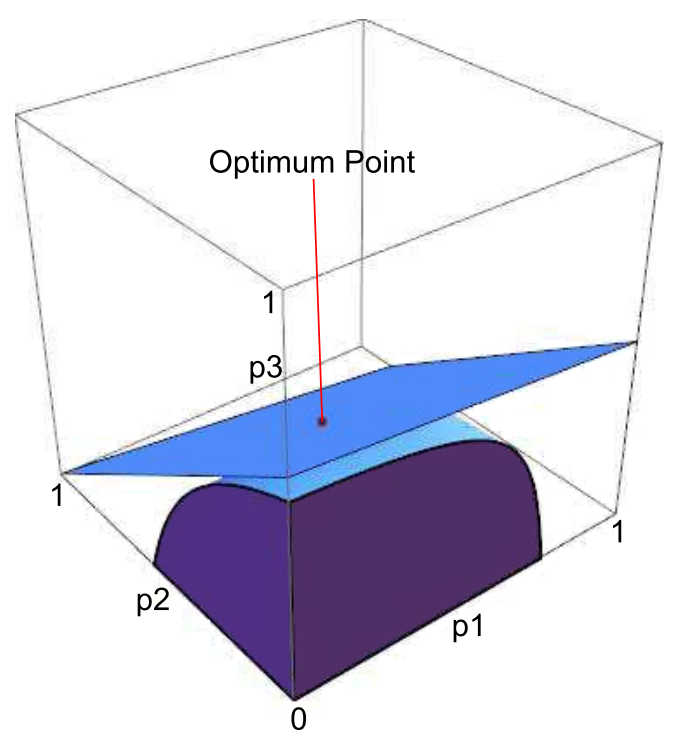

Figure 2: (Color online)The optimum point is a non-singular interior point of the critical feasible region.

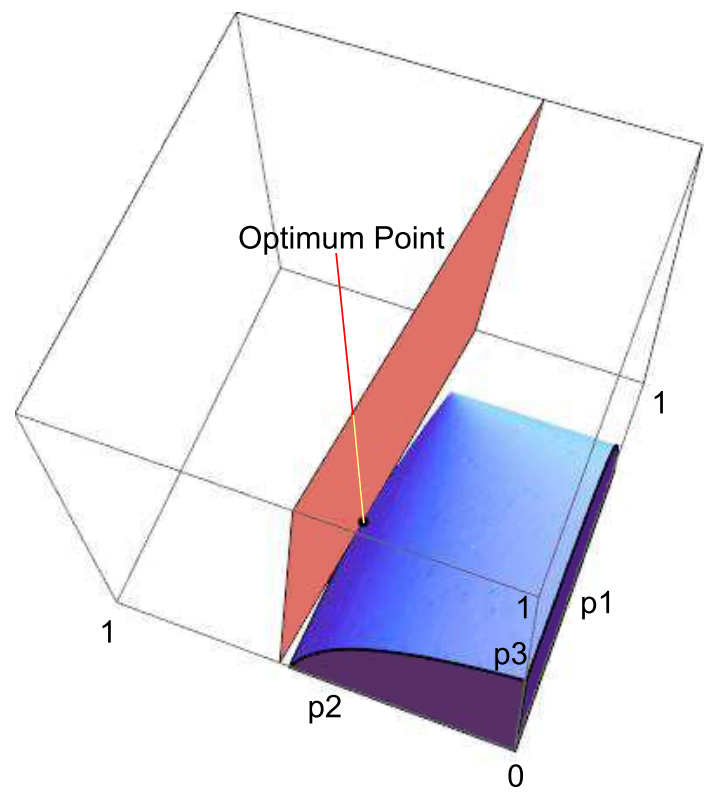

Figure 3: (Color online) The optimum point is on the boundary $\mathcal{B}_{R}$.

where $\Pi_{0}$ is defined in Eq. (6). Define an $n \times n$ matrix $C$ with $\left|\phi_{k}\right\rangle$ as its $k$ th column,

$$
C=\left(\left|\phi_{1}\right\rangle, \cdots,\left|\phi_{n}\right\rangle\right) \text {. }
$$

It can be directly verified that

$$
X-\Gamma=C^{\dagger} C,
$$

using Eq. (1).

Before presenting the main theorem of this section, we give three lemmas that will be used later as follows.

Lemma 4. Suppose $A$ is an $n \times n$ matrix. Let the adjugate of $A$ be denoted by $A^{*}$, whose $(i, j)$ entry is the $(j, i)$ cofactor of $A$. Then

$$
\operatorname{Rank} A^{*}= \begin{cases}n & \text { if } \operatorname{Rank} A=n \\ 1 & \text { if } \operatorname{Rank} A=n-1 \\ 0 & \text { if } \operatorname{Rank} A \leq n-2\end{cases}
$$




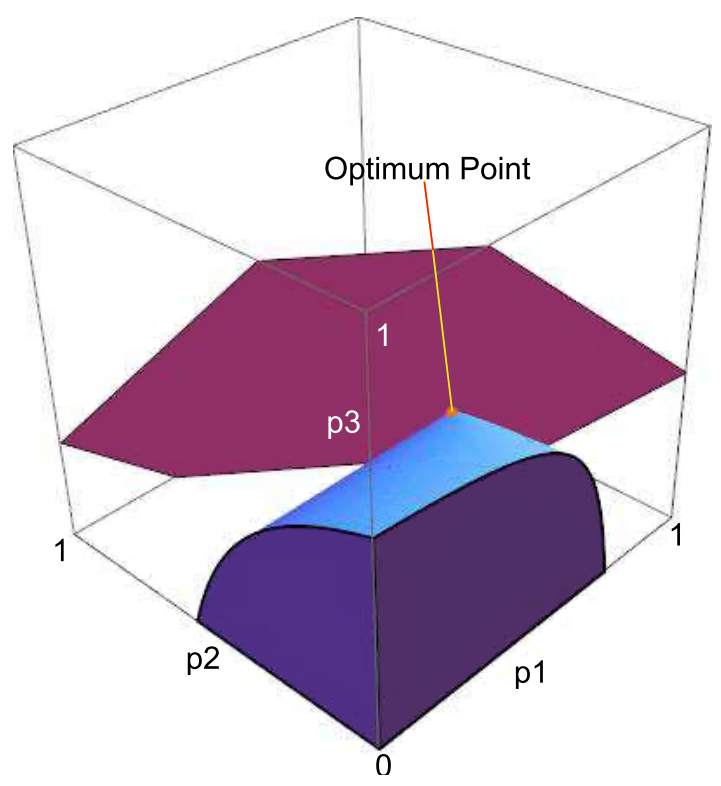

Figure 4: (Color online) The optimum point is a singular point.

Lemma 5(Binet-Cauchy). Suppose $A$ and $B$ are matrices of sizes $m \times n$ and $n \times m$ respectively, then

$$
\operatorname{det}(A B)=\left\{\begin{array}{cl}
0 & \text { if } m>n \\
\operatorname{det} A \cdot \operatorname{det} B & \text { if } m=n \\
\sum_{1 \leq j_{1}<\cdots<j_{m} \leq n} \operatorname{det} A\left(\begin{array}{ccc}
1 & \cdots & m \\
j_{1} & \cdots & j_{m}
\end{array}\right) \operatorname{det} B\left(\begin{array}{ccc}
j_{1} & \cdots & j_{m} \\
1 & \cdots & m
\end{array}\right) & \text { if } m<n
\end{array} .\right.
$$

where $A\left(\begin{array}{ccc}1 & \cdots & m \\ j_{1} & \cdots & j_{m}\end{array}\right)$ denotes the $m \times m$ submatrix of $A$ whose $k$ th column is the $j_{k}$ th column of $A$, and $B\left(\begin{array}{ccc}j_{1} & \cdots & j_{m} \\ 1 & \cdots & m\end{array}\right)$ denotes the $m \times m$ submatrix of $B$ whose $k$ th row is the $j_{k}$ th row of $B$.

Proof of these two lemmas can be found in many algebra text books.

Lemma 6. Let $C^{*}$ denote the adjugate of the matrix $C$ defined in Eq. (51) and $\left|c_{k}\right\rangle$ denote the transpose of the $k$ th row of $C^{*}(k=1, \cdots, n)$. If the optimum point $\boldsymbol{p}_{\text {opt }}$ is a non-singular interior point in the critical feasible region, then at $\boldsymbol{p}_{\text {opt }}$ each $\left|c_{k}\right\rangle$ can be written as

$$
\left.\left|c_{k}\right\rangle\right|_{\boldsymbol{p}_{\text {opt }}}=\sqrt{\gamma_{k}} \xi e^{i \theta_{k}}|\rho\rangle,
$$

where $|\rho\rangle$ is some normalized vector, $\xi$ is a positive parameter and $e^{i \theta_{k}}$ is a phase to be determined.

Proof. Let $C_{k}$ denote the submatrix of $C$ by deleting the $k$ th column $\left|\phi_{k}\right\rangle$, and $(X-\Gamma)_{i j}$ denote the submatrix of $X-\Gamma$ by deleting the $i$ th row and the $j$ th column. Similar to Eq. (52), it can be directly verified that

$$
(X-\Gamma)_{i j}=C_{i}^{\dagger} C_{j}
$$

so

$$
\operatorname{det}(X-\Gamma)_{i j}=\operatorname{det}\left(C_{i}^{\dagger} C_{j}\right)=(-1)^{i+j}\left\langle c_{i} \mid c_{j}\right\rangle
$$

according to Lemma 5 ,

Since $\boldsymbol{p}_{\text {opt }}$ is a non-singular interior point in the critical feasible region $\mathcal{R}_{C F}$, we have $M_{k}\left(\boldsymbol{p}_{\text {opt }}\right) \neq 0$ for some $k \in\{1, \cdots, n\}$, which implies that $\left.\operatorname{Rank}(X-\Gamma)\right|_{p_{o p t}}=n-1$, or Rank $\left.C\right|_{p_{o p t}}=n-1$, equivalently. Therefore, Rank $\left.C^{*}\right|_{p_{\text {opt }}}=1$ according to Lemma 4, implying that all $\left|c_{k}\right\rangle$ 's are proportional to each other. So each $\left|c_{k}\right\rangle$ at $\boldsymbol{p}_{\text {opt }}$ can be written as

$$
\left.\left|c_{k}\right\rangle\right|_{p_{o p t}}=a_{k}|\rho\rangle
$$


where $|\rho\rangle$ is some normalized vector and $a_{k}$ is a coefficient to be determined.

From Eq. (57), we have

$$
M_{k}(\boldsymbol{p})=\operatorname{det}(X-\Gamma)_{k k}=\left\langle c_{k} \mid c_{k}\right\rangle .
$$

By substituting Eqs. (25) and (58) into (59), we have

$$
a_{k}^{*} a_{k}=\gamma_{k} \lambda
$$

where $\lambda>0$. Let $\lambda=\xi^{2}$, then Eq. (55) holds.

The factor $\xi$ cannot be zero, otherwise $\sigma_{n}(\boldsymbol{p})=0$ would be degenerate at $\boldsymbol{p}_{\text {opt }}$, contradicting the assumption that $\boldsymbol{p}_{\text {opt }}$ is not a singular point.

When the states to be discriminated and the prior probabilities are fixed, the difference between any pair of phase factors $e^{i \theta_{i}}, e^{i \theta_{j}}(i \neq j)$ is fixed, while the phase factors $e^{i \theta_{k}}(k=1, \cdots, n)$ themselves can be altered since an arbitrary total phase can always be added to all $e^{i \theta_{k}}$ 's by choosing an appropriate phase for $|\rho\rangle$. The phase differences are what really matter in the following discussion.

Theorem 5. If the optimum point $\boldsymbol{p}_{\text {opt }}$ is a non-singular point in the interior part $\Omega_{R}$ of the critical feasible region, then the components of $\boldsymbol{p}_{\text {opt }}$ can be written as

$$
p_{i}=e^{-i \theta_{i}} \sum_{k=1}^{n} e^{i \theta_{k}} \sqrt{\frac{\gamma_{k}}{\gamma_{i}}}\left\langle\psi_{i} \mid \psi_{k}\right\rangle, \quad \forall i=1, \cdots, n
$$

and the optimum average success probability can also be written as

$$
\bar{p}_{\text {opt }}=\| \sum_{k=1}^{n} \sqrt{\gamma_{k}} e^{i \theta_{k}}\left|\psi_{k}\right\rangle \|^{2}
$$

Proof. Since $\boldsymbol{p}_{\text {opt }}$ is a non-singular interior point in the critical feasible region, by substituting Eq. (55) in Lemma 6 into (57), we get

$$
\left.\operatorname{det}\left(C_{i}^{\dagger} C_{k}\right)\right|_{\boldsymbol{p}_{\text {opt }}}=(-1)^{i+k} \sqrt{\gamma_{i} \gamma_{k}} e^{-i \theta_{i}+i \theta_{k}} \xi^{2}, \forall i, k=1, \cdots, n
$$

Noting that the $(i, j)$ entry of $X-\Gamma$ is $\left\langle\psi_{i} \mid \psi_{j}\right\rangle-p_{i} \delta_{i j}$, the algebraic cofactor of the $(i, j)$ entry is $(-1)^{i+j}$ det $(X-\Gamma)_{i j}$ and $\operatorname{det}(X-\Gamma)=0$, we perform Laplace expansion on the determinant of $X-\Gamma$ along its $i$ th row,

$$
\begin{aligned}
\left.\operatorname{det}(X-\Gamma)\right|_{\boldsymbol{p}_{\text {opt }}} & =\left.\sum_{k=1}^{n}(-1)^{i+k}\left\langle\psi_{i} \mid \psi_{k}\right\rangle \operatorname{det}(X-\Gamma)_{i k}\right|_{\boldsymbol{p}_{\text {opt }}}-p_{i} M_{i}\left(\boldsymbol{p}_{\text {opt }}\right) \\
& =\left.\sum_{k=1}^{n}(-1)^{i+k}\left\langle\psi_{i} \mid \psi_{k}\right\rangle \operatorname{det}\left(C_{i}^{\dagger} C_{k}\right)\right|_{\boldsymbol{p}_{\text {opt }}}-p_{i} M_{i}\left(\boldsymbol{p}_{\text {opt }}\right) \\
& =\sum_{k=1}^{n}\left\langle\psi_{i} \mid \psi_{k}\right\rangle \sqrt{\gamma_{i} \gamma_{k}} e^{-i \theta_{i}+i \theta_{k}} \xi^{2}-\gamma_{i} p_{i} \xi^{2}=0
\end{aligned}
$$

for $i=1, \cdots, n$, where we have used Eqs. (57), (59) and (63). Eliminating $\xi^{2}$ from both sides of (64), we obtain

$$
\gamma_{i} p_{i}=\sum_{k=1}^{n}\left\langle\psi_{i} \mid \psi_{k}\right\rangle \sqrt{\gamma_{i} \gamma_{k}} e^{-i \theta_{i}+i \theta_{k}}=\sqrt{\gamma_{i}} e^{-i \theta_{i}}\left\langle\psi_{i}\left|\sum_{k=1}^{n} \sqrt{\gamma_{k}} e^{i \theta_{k}}\right| \psi_{k}\right\rangle, \quad \forall i, k=1, \cdots, n
$$

which immediately implies Eq. (61). Summing up Eq. (65) for all $i=1, \cdots, n$, we eventually get Eq. (62).

It should be pointed out that a similar result was derived in Ref. [38] for the special case where $X$ is a real matrix, and our Theorem 5 can be considered as a generalization of that result to the situation where $X$ is complex.

It should also be mentioned that Theorem 5 actually gives an analytical relation between the maximum average success probability and the $n$ pure states to be discriminated but not a complete analytical solution, since the explicit expressions of the phases $e^{i \theta_{k}}(k=1, \cdots, n)$ are not given in Theorem 5 . However, Theorem 5 may sometimes help to simplify the calculation of the optimum solution in special cases, as we shall show in the next subsection. And it may also help to obtain some bounds of $\bar{p}_{\text {opt }}$ or work out the phases $e^{i \theta_{k}}(k=1, \cdots, n)$ by numerical methods according to Remark 3 given later. 
Remark 2. It seems that the solution (61) and (62) would change if the phase of any state $\left|\psi_{k}\right\rangle$ is changed. But actually the corresponding $e^{i \theta_{k}}$ will also be changed in that case and we can see below that any term $e^{i \theta_{k}}\left|\psi_{k}\right\rangle$ in Eqs. (61) and (62) remains unchanged up to a global phase for all $e^{i \theta_{k}}\left|\psi_{k}\right\rangle$ 's.

In fact, if some $\left|\psi_{i}\right\rangle$ is transformed as

$$
\left|\psi_{i}\right\rangle \rightarrow e^{i \chi}\left|\psi_{i}\right\rangle
$$

where $e^{i \chi}$ is an arbitrary phase while the other $n-1$ states stay unchanged, then according to the definitions of $\left|c_{k}\right\rangle$ and $e^{i \theta_{k}}$ in Lemma 6, the phase $e^{i \theta_{k}}$ changes as follows:

$$
e^{i \theta_{k}} \rightarrow\left\{\begin{array}{ll}
e^{i\left(\theta_{k}+\chi\right)} & \text { if } k \neq i \\
e^{i \theta_{k}} \text { (unchanged) } & \text { if } k=i
\end{array} .\right.
$$

Since any global phase can be eliminated from all $e^{i \theta_{k}}$ 's by the vector $|\rho\rangle$, Eq. (67) is equivalent to

$$
e^{i \theta_{k}} \rightarrow\left\{\begin{array}{ll}
e^{i \theta_{k}} \text { (unchanged) } & \text { if } k \neq i \\
e^{i\left(\theta_{k}-\chi\right)} & \text { if } k=i
\end{array} .\right.
$$

Therefore any term $e^{i \theta_{k}}\left|\psi_{k}\right\rangle$ (including the one that $k=i$ ) stays unchanged, considering Eq. (66).

Remark 3. The maximum average probability has an interesting property that it must be the value of a stationary point [64] of the expression at the right side of Eq. (62) if the phases $e^{i \theta_{k}}$ are allowed to change freely. This is because $p_{i}$ is real which requires that

$$
\sqrt{\gamma_{i}} e^{-i \theta_{i}}\left\langle\psi_{i}\right|\left(\sum_{k=1}^{n} \sqrt{\gamma_{k}} e^{i \theta_{k}}\left|\psi_{k}\right\rangle\right)-\left(\sum_{k=1}^{n} \sqrt{\gamma_{k}} e^{-i \theta_{k}}\left\langle\psi_{k}\right|\right) \sqrt{\gamma_{i}} e^{i \theta_{i}}\left|\psi_{i}\right\rangle=0
$$

according to Eq. (61), and Eq. (69) is equivalent to

$$
\frac{\partial}{\partial \theta_{i}} \| \sum_{k=1}^{n} \sqrt{\gamma_{k}} e^{i \theta_{k}}\left|\psi_{k}\right\rangle \|^{2}=0
$$

which is exactly the restriction equation of $\theta_{i}$ that must be satisfied when the expression at the right side of (62) reaches a stationary point.

\section{B. Example}

In this subsection, we give an example to show the use of Theorem 5 .

Suppose $\left\{\left|\psi_{i}\right\rangle\right\}_{i=1}^{n}$ is a set of linearly independent pure states that $\left\langle\psi_{1} \mid \psi_{i}\right\rangle \neq 0, i=2, \cdots, n$ and $\left\langle\psi_{i} \mid \psi_{j}\right\rangle=0$, $\forall i, j=2, \cdots, n$, let's calculate the maximum average success probability when the optimum point $\boldsymbol{p}_{\text {opt }}$ is a nonsingular interior point in the critical feasible region $\mathcal{R}_{C F}$.

Without loss of generality, we can choose that $e^{i \theta_{1}}=1$. According to Eq. (61) and the fact that $p_{i}$ is real $(i=1, \cdots, n)$, we can directly get

$$
\theta_{k}=\operatorname{Arg}\left(\left\langle\psi_{k} \mid \psi_{1}\right\rangle\right)-\pi, \quad(k=2, \cdots, n)
$$

SO

$$
\left\{\begin{array}{l}
p_{k}=1-\sqrt{\frac{\gamma_{1}}{\gamma_{k}}}\left|\left\langle\psi_{k} \mid \psi_{1}\right\rangle\right| \quad(k=2, \cdots, n) \\
p_{1}=1-\sum_{k=2}^{n} \sqrt{\frac{\gamma_{k}}{\gamma_{1}}}\left|\left\langle\psi_{1} \mid \psi_{k}\right\rangle\right|
\end{array} .\right.
$$

Substituting Eq. (171) into (62) or using $\bar{p}=\sum_{i=1}^{n} \gamma_{i} p_{i}$, we have

$$
\bar{p}_{\text {opt }}=\sum_{k=1}^{n} \gamma_{k} p_{k}=1-2 \sum_{k=2}^{n} \sqrt{\gamma_{1} \gamma_{k}}\left|\left\langle\psi_{k} \mid \psi_{1}\right\rangle\right| \text {. }
$$

Since $0 \leq p_{k} \leq 1, k=1, \cdots, n$, according to Eq. (172), we obtain $\sqrt{\frac{\gamma_{k}}{\gamma_{1}}} \geq\left|\left\langle\psi_{k} \mid \psi_{1}\right\rangle\right|($ for all $k=2, \cdots, n)$ and $\sum_{k=2}^{n} \sqrt{\frac{\gamma_{k}}{\gamma_{1}}}\left|\left\langle\psi_{1} \mid \psi_{k}\right\rangle\right| \leq 1$, which are the conditions for the optimum point $\boldsymbol{p}_{\text {opt }}$ to be a non-singular interior point in the critical feasible region $\mathcal{R}_{C F}$.

When $n=2$, Eq. (73) gives the well known Ivanovic-Dieks-Peres limit [27, 28, 29, 30]. 


\section{A GENERALIZED EQUAL-PROBABILITY MEASUREMENT PROBLEM}

A special scheme to discriminate quantum states unambiguously is the so-called equal-probability measurement (EPM) [56], which requires that the probability of each measurement outcome is equal, i.e., $p_{1}=\cdots=p_{n}$. In this section we will treat a generalized version of the EPM problem, which is defined as follows.

The Generalized EPM Problem (GEPM). If it is required that

$$
p_{1}: p_{2}: \cdots: p_{n}=w_{1}: w_{2}: \cdots: w_{n}
$$

for a given set of non-negative numbers $w_{i}\left(w_{i} \geq 0, \forall i=1, \cdots, n\right)$ when the average success probability of unambiguously discriminating the states $\left\{\left|\psi_{i}\right\rangle\right\}_{i=1}^{n}$ reaches the maximum, one needs to work out the prior probabilities $\left\{\gamma_{i}\right\}_{i=1}^{n}$ or the conditions these prior probabilities should satisfy.

We have the following result on this generalized version of EPM problem using Theorem 3

Theorem 6(Generalized EPM). Suppose $\Psi$ is a matrix with $\left|\psi_{i}\right\rangle / \sqrt{w_{i}}$ as its $i$ th column, let $\sigma_{m i n}$ denote the minimum eigenvalue of $\Psi^{\dagger} \Psi$ and $M_{i}^{G E P M}=\left.M_{i}(\boldsymbol{p})\right|_{p_{1}=w_{1} \sigma_{\min }, \cdots, p_{n}=w_{n} \sigma_{\min }}$ be $(n-1) \times(n-1)$ principal minor of $X-\Gamma$ corresponding to its $i$ th diagonal element while $p_{1}=w_{1} \sigma_{\min }, \cdots, p_{n}=w_{n} \sigma_{\min }$. If $M_{i}^{G E P M}>0$ for some $i \in$ $\{1, \cdots, n\}$, then the sufficient and necessary conditions that the GEPM is the optimum POVM to unambiguously discriminate the given states is that

$$
\gamma_{i}=\frac{M_{i}^{G E P M}}{\sum_{i=1}^{n} M_{i}^{G E P M}}, \quad \forall i=1, \cdots, n .
$$

If $M_{i}^{G E P M}=0$ for all $i \in\{1, \cdots, n\}$, then there exist a range of different $\gamma$ 's for which the GEPM is the optimum POVM for unambiguous discrimination of the states $\left\{\left|\psi_{i}\right\rangle\right\}_{i=1}^{n}$.

Proof. We suppose that the optimum solution $\boldsymbol{p}_{\text {opt }}$ is $p_{1}=w_{1} \eta, \cdots, p_{n}=w_{n} \eta$ according to Eq. (74), and the corresponding $\Gamma$ matrix is $\operatorname{diag}\left(w_{1} \eta, w_{2} \eta, \cdots, w_{n} \eta\right)$, where $\eta$ is to be determined. By some simple calculation, it can be shown that in this situation the condition $X-\Gamma \geq 0$ can be converted to $\Psi^{\dagger} \Psi-\eta I \geq 0$ where $I$ is the identity matrix, so $\sigma_{\min }$, the minimum eigenvalue of $\Psi^{\dagger} \Psi$, is exactly the maximum feasible value of $\eta$, which means that $p_{i}=w_{i} \sigma_{\min }(i=1, \cdots, n)$ is the optimum solution $\boldsymbol{p}_{\text {opt }}$ when Eq. (74) has to be satisfied. If $M_{i}^{G E P M} \neq 0$ for some $i \in\{1, \cdots, n\}, \boldsymbol{p}_{\text {opt }}$ is not a singular point, so Eq. (75) holds according to Eq. (25).

On the other hand, if $M_{i}^{G E P M}=0$ for all $i \in\{1, \cdots, n\}$, then the point where $p_{1}=w_{1} \sigma_{\text {min }}, \cdots, p_{n}=w_{n} \sigma_{m i n}$ in the critical feasible region is a singular point, so the normal vector changes discontinuously in the neighborhood of $\boldsymbol{p}=\left(w_{1} \sigma_{\text {min }}, \cdots, w_{n} \sigma_{\text {min }}\right)$ in the critical feasible region $\mathcal{R}_{C F}$. Thus, the GEPM is the optimum unambiguous discrimination scheme for a range of different $\gamma$ 's.

The solution of the original EPM problem follows immediately from Theorem 6 by setting $w_{1}=\cdots=w_{n}=1$.

It is obvious that for any set of linearly independent quantum states, it is always possible to find prior probabilities $\left\{\gamma_{1}, \cdots, \gamma_{n}\right\}$ such that the generalized EPM is the optimum scheme to unambiguously discriminate these states.

\section{UNAMBIGUOUS DISCRIMINATION OF THREE PURE STATES}

In this section we shall use the results and method presented in Sec. III to study the unambiguous discrimination problem of three linearly independent pure states, mainly for the non-singular interior optimum solution.

\section{A. General equations}

Suppose the three states to be discriminated are $\left|\psi_{1}\right\rangle,\left|\psi_{2}\right\rangle,\left|\psi_{3}\right\rangle$ with prior probabilities $\gamma_{1}, \gamma_{2}, \gamma_{3}$, and they are linearly independent. Then according to Eq. (25) we can have the following equations

$$
\begin{aligned}
& M_{1}(\boldsymbol{p})=\left(1-p_{2}\right)\left(1-p_{3}\right)-\left|\left\langle\psi_{2} \mid \psi_{3}\right\rangle\right|^{2}=\lambda \gamma_{1}, \\
& M_{2}(\boldsymbol{p})=\left(1-p_{1}\right)\left(1-p_{3}\right)-\left|\left\langle\psi_{1} \mid \psi_{3}\right\rangle\right|^{2}=\lambda \gamma_{2}, \\
& M_{3}(\boldsymbol{p})=\left(1-p_{1}\right)\left(1-p_{2}\right)-\left|\left\langle\psi_{1} \mid \psi_{2}\right\rangle\right|^{2}=\lambda \gamma_{3} .
\end{aligned}
$$


From these three equations, we can obtain

$$
\begin{aligned}
& 1-p_{1}=\sqrt{\frac{\left(\left|\left\langle\psi_{1} \mid \psi_{2}\right\rangle\right|^{2}+\lambda \gamma_{3}\right)\left(\left|\left\langle\psi_{1} \mid \psi_{3}\right\rangle\right|^{2}+\lambda \gamma_{2}\right)}{\left|\left\langle\psi_{2} \mid \psi_{3}\right\rangle\right|^{2}+\lambda \gamma_{1}}}, \\
& 1-p_{2}=\sqrt{\frac{\left(\left|\left\langle\psi_{2} \mid \psi_{3}\right\rangle\right|^{2}+\lambda \gamma_{1}\right)\left(\left|\left\langle\psi_{1} \mid \psi_{2}\right\rangle\right|^{2}+\lambda \gamma_{3}\right)}{\left|\left\langle\psi_{1} \mid \psi_{3}\right\rangle\right|^{2}+\lambda \gamma_{2}}}, \\
& 1-p_{3}=\sqrt{\frac{\left(\left|\left\langle\psi_{2} \mid \psi_{3}\right\rangle\right|^{2}+\lambda \gamma_{1}\right)\left(\left|\left\langle\psi_{1} \mid \psi_{3}\right\rangle\right|^{2}+\lambda \gamma_{2}\right)}{\left|\left\langle\psi_{1} \mid \psi_{2}\right\rangle\right|^{2}+\lambda \gamma_{3}}} .
\end{aligned}
$$

Substituting Eqs. (799), (80) and (81) into $\operatorname{det}(X-\Gamma)=0$ and making some rearrangements, we can get the following equation of $\lambda$

$$
\gamma \lambda^{3}-S \lambda-2|T|^{2}+2 \sqrt{\gamma \lambda^{3}+R \lambda^{2}+S \lambda+|T|^{2}} \operatorname{Re}(T)=0
$$

where $\operatorname{Re}(T)$ represents the real part of $T$ and $S, T, R, \gamma$ are defined as

$$
\begin{gathered}
\gamma=\gamma_{1} \gamma_{2} \gamma_{3}, \quad T=\left\langle\psi_{1} \mid \psi_{2}\right\rangle\left\langle\psi_{2} \mid \psi_{3}\right\rangle\left\langle\psi_{3} \mid \psi_{1}\right\rangle, \\
R=\gamma_{1} \gamma_{2}\left|\left\langle\psi_{1} \mid \psi_{2}\right\rangle\right|^{2}+\gamma_{2} \gamma_{3}\left|\left\langle\psi_{2} \mid \psi_{3}\right\rangle\right|^{2}+\gamma_{1} \gamma_{3}\left|\left\langle\psi_{1} \mid \psi_{3}\right\rangle\right|^{2}, \\
S=\gamma_{1}\left|\left\langle\psi_{1} \mid \psi_{2}\right\rangle\right|^{2}\left|\left\langle\psi_{3} \mid \psi_{1}\right\rangle\right|^{2}+\gamma_{2}\left|\left\langle\psi_{1} \mid \psi_{2}\right\rangle\right|^{2}\left|\left\langle\psi_{2} \mid \psi_{3}\right\rangle\right|^{2}+\gamma_{3}\left|\left\langle\psi_{2} \mid \psi_{3}\right\rangle\right|^{2}\left|\left\langle\psi_{3} \mid \psi_{1}\right\rangle\right|^{2} .
\end{gathered}
$$

In Ref. [31], it is doubted that whether a general closed form of the maximum average success probability of unambiguous state discrimination exists. One can show that Eq. (82) can be converted to a polynomial equation of degree 6 , so it is generally difficult to find an analytical solution for unambiguous discrimination of three states. However, in some special situations, analytical solutions can be obtained, and we will give some examples in the next two subsections.

\section{B. Special cases}

For some special cases, Eq. (82) can be simplified and one can obtain exact analytical solutions.

Case 1. Suppose that $\left\langle\psi_{1} \mid \psi_{2}\right\rangle=0$, but $\left\langle\psi_{2} \mid \psi_{3}\right\rangle \neq 0,\left\langle\psi_{3} \mid \psi_{1}\right\rangle \neq 0$, then Eq. (82) can be simplified to

$$
\gamma_{1} \gamma_{2} \gamma_{3} \lambda^{3}-\left|\left\langle\psi_{3} \mid \psi_{1}\right\rangle\left\langle\psi_{2} \mid \psi_{3}\right\rangle\right|^{2} \gamma_{3} \lambda=0
$$

We can easily obtain that $\lambda=0$ or

$$
\lambda=\frac{\left|\left\langle\psi_{3} \mid \psi_{1}\right\rangle\left\langle\psi_{2} \mid \psi_{3}\right\rangle\right|}{\sqrt{\gamma_{1} \gamma_{2}}},
$$

and the negative root has been discarded.

With some observation, it can be verified that $\lambda=0$ is not a solution for Eqs. (79)-(81), unless $\left\langle\psi_{1} \mid \psi_{3}\right\rangle=0$ or $\left\langle\psi_{2} \mid \psi_{3}\right\rangle=0$, which is a trivial case. Thus Eq. (87) is the unique solution for this situation. Substituting Eq. (87) into (79)- (81), we can get the optimum average probability

$$
\bar{p}_{\text {opt }}=1-2 \sqrt{\gamma_{1} \gamma_{3}}\left|\left\langle\psi_{1} \mid \psi_{3}\right\rangle\right|-2 \sqrt{\gamma_{2} \gamma_{3}}\left|\left\langle\psi_{2} \mid \psi_{3}\right\rangle\right|
$$

This agrees with the result in Sec. IVB.

Case 2. Suppose that $\left\langle\psi_{1} \mid \psi_{2}\right\rangle\left\langle\psi_{2} \mid \psi_{3}\right\rangle\left\langle\psi_{3} \mid \psi_{1}\right\rangle$ is purely imaginary, i.e. $\operatorname{Re}\left(\left\langle\psi_{1} \mid \psi_{2}\right\rangle\left\langle\psi_{2} \mid \psi_{3}\right\rangle\left\langle\psi_{3} \mid \psi_{1}\right\rangle\right)=0$, but $\left\langle\psi_{1} \mid \psi_{2}\right\rangle\left\langle\psi_{2} \mid \psi_{3}\right\rangle\left\langle\psi_{3} \mid \psi_{1}\right\rangle \neq 0$, then Eq. (82) becomes

$$
\gamma \lambda^{3}-S \lambda-2|T|^{2}=0
$$


This is a cubic equation of $\lambda$, so we can get analytical solutions in general. It can be proved that this equation has and only has one positive root of $\lambda$ using Vieta's theorem [69], and this positive root is

$$
\lambda=\frac{\left(27 \gamma^{2}|T|^{2}+3 \sqrt{81 \gamma^{4}|T|^{4}-3 \gamma^{3} S^{3}}\right)^{\frac{1}{3}}}{3 \gamma}+\frac{S}{\left(27 \gamma^{2}|T|^{2}+3 \sqrt{81 \gamma^{4}|T|^{4}-3 \gamma^{3} S^{3}}\right)^{\frac{1}{3}}},
$$

where we have used the notations defined in Eqs. 83)-(85).

It can be shown that

$$
S^{3} \geq 27 \gamma|T|^{4}
$$

by the mean inequality [70], so Eq. (20) can be simplified to

$$
\lambda=2 \sqrt{\frac{S}{3 \gamma}} \cos \frac{\theta}{3}
$$

where

$$
\theta=\arccos \frac{|T|^{2}}{S} \sqrt{\frac{27 \gamma}{S}} .
$$

Substituting Eq. (92) into (779)-(81), we can eventually get $p_{1}, p_{2}, p_{3}$ and $\bar{p}$ for the optimum solution.

Case 3. If $\left\langle\psi_{1} \mid \psi_{2}\right\rangle\left\langle\psi_{2} \mid \psi_{3}\right\rangle\left\langle\psi_{3} \mid \psi_{1}\right\rangle$ is real and non-negative, then it can be directly verified that $\lambda=0$ is a solution to Eq. (82), which implies that a singular point exists in the critical feasible region. And in this case, Eq. (82) can be simplified to

$$
\lambda^{2}\left(\gamma^{2} \lambda^{4}-2 \gamma S \lambda^{2}-8 \gamma|T|^{2} \lambda-Q\right)=0,
$$

where in addition to Eqs. (83)-(85), another constant $Q$ is defined as

$$
Q=S^{2}-4 R|T|^{2} \text {. }
$$

Since the expression inside the parentheses of Eq. (94) is a quadratic polynomial, analytical solutions of $\lambda$ can be obtained from (94). But the analytical solution is too complicated to show any practical meaning, so we are not going to include it here.

It should be pointed out that the analytical solutions for three pure states with with real Gram matrix was given in Ref. [38], and our Case 3 generalizes that result.

\section{Equal-Probability Measurement}

In Sec. $\mathrm{V}$, we have given the exact solutions of the prior probabilities to the generalized EPM problem. As an example, we solve the original EPM problem of three pure states in a direct way.

Let $p_{1}=p_{2}=p_{3}=p_{E P M}$ and substitute it into $\operatorname{det}(X-\Gamma)=0$, one can get

$$
p_{E P M}=1-\frac{(1+3 i) W}{2 \cdot 3^{\frac{1}{3}}\left(\sqrt{3} \sqrt{27(\operatorname{Re} T)^{2}-W^{3}}-9 \operatorname{Re} T\right)^{\frac{1}{3}}}-\frac{(1-3 i)\left(\sqrt{3} \sqrt{27(\operatorname{Re} T)^{2}-W^{3}}-9 \operatorname{Re} T\right)^{\frac{1}{3}}}{2 \cdot 3^{2 / 3}},
$$

where $T$ is defined in Eq. (83) and

$$
W=\left|\left\langle\psi_{1} \mid \psi_{2}\right\rangle\right|^{2}+\left|\left\langle\psi_{1} \mid \psi_{3}\right\rangle\right|^{2}+\left|\left\langle\psi_{2} \mid \psi_{3}\right\rangle\right|^{2} .
$$

It can be verified that

$$
27(\operatorname{Re} T)^{2} \leq 27|T|^{2} \leq W^{3}
$$


where the second inequality can be proved by the mean inequality [70], then $p_{E P M}$ can be reduced to

$$
p_{E P M}=1-2 \cdot \sqrt{\frac{W}{3}} \cos \left(\frac{\pi}{3}-\frac{\theta}{3}\right),
$$

where

$$
\theta=\arccos \frac{3 \sqrt{3} \operatorname{Re}(T)}{W \sqrt{W}} .
$$

Thus according to Eqs. (76) $-(78)$, the prior probabilities $\gamma_{1}, \gamma_{2}, \gamma_{3}$ must be

$$
\left\{\begin{array}{l}
\gamma_{1}=\frac{\frac{4}{3} W \cos ^{2}\left(\frac{\pi}{3}-\frac{\theta}{3}\right)-\left|\left\langle\psi_{2} \mid \psi_{3}\right\rangle\right|^{2}}{4 W \cos ^{2}\left(\frac{\pi}{3}-\frac{\theta}{3}\right)-W} \\
\gamma_{2}=\frac{\frac{4}{3} W \cos ^{2}\left(\frac{\pi}{3}-\frac{\theta}{3}\right)-\left|\left\langle\psi_{1} \mid \psi_{3}\right\rangle\right|^{2}}{4 W \cos ^{2}\left(\frac{\pi}{3}-\frac{\theta}{3}\right)-W} \\
\gamma_{3}=\frac{\frac{4}{3} W \cos ^{2}\left(\frac{\pi}{3}-\frac{\theta}{3}\right)-\left|\left\langle\psi_{1} \mid \psi_{2}\right\rangle\right|^{2}}{4 W \cos ^{2}\left(\frac{\pi}{3}-\frac{\theta}{3}\right)-W}
\end{array}\right.
$$

Remark 4. In Eq. (101), the denominators of $\gamma_{1}, \gamma_{2}, \gamma_{3}$ can be equal to zero, and in this situation the optimum EPM point is a singular point. This can be shown as follows. If the dominators in (101) are equal to zero, then

$$
\cos \left(\frac{\pi}{3}-\frac{\theta}{3}\right)=\frac{1}{2}
$$

so

$$
\theta=0 \text { or } 2 \pi
$$

(Note that $\cos \left(\frac{\pi}{3}-\frac{\theta}{3}\right)$ can not be $-\frac{1}{2}$, otherwise $p_{E P M}$ would be larger than 1 according to Eq. (99).)

Then it can be seen that

$$
3 \sqrt{3} \operatorname{Re}(T)=W \sqrt{W}
$$

by substituting Eq. (103) into (100).

According to Eq. (98) and the condition that "=" holds in a mean inequality [70], one can have

$$
\left|\left\langle\psi_{1} \mid \psi_{2}\right\rangle\right|=\left|\left\langle\psi_{1} \mid \psi_{3}\right\rangle\right|=\left|\left\langle\psi_{2} \mid \psi_{3}\right\rangle\right| \text {. }
$$

Then according to Eqs. (97) and (99),

$$
p_{1}=p_{2}=p_{3}=p_{E P M}=1-\left|\left\langle\psi_{1} \mid \psi_{2}\right\rangle\right| .
$$

Substituting Eq. (106) into Eqs. (76)-(78), it can be seen that

$$
M_{1}(\boldsymbol{p})=M_{2}(\boldsymbol{p})=M_{3}(\boldsymbol{p})=0 .
$$

So, when the dominators in Eq. (101) are equal to zero, the optimum EPM point is a singular point.

\section{CONCLUSION}

In this paper, we have mainly studied the problem of optimum unambiguous discrimination of $n$ linearly independent pure states. We have derived some analytical properties of the optimum solution to this problem, and established two sets of new equations in Theorems 3 and 4 which provide detailed methods to obtain the optimum solution in different situations. We have also presented a geometrical illustration of the equations we established with a numerical example in Sec. IIIB. An analytical formula which shows the relation between the optimum solution of the unambiguous discrimination problem and the $n$ pure states to be identified has been derived in Sec. IV. And we have also solved a generalized EPM problem in Sec. V] with the proportion of the occurring probabilities of the measurement outcomes to be fixed. Finally, the optimum unambiguous discrimination problem of three pure states is studied, and analytical results has been presented for some interesting cases in Sec. VI.

It is no doubt that the problem of discriminating quantum states is important in quantum information science since it has wide application to quantum cryptography and quantum communication, so it motivates a lot of researchers to explore different kinds of optimum discrimination strategies. In addition to many important results mentioned in Sec. I. some other novel strategies such as minimax discrimination [65, 66] and maximum confidence discrimination [67, 68] have been introduced recently. We hope that our results presented in this article may stimulate further research to the optimum state discrimination problem in general. 


\section{Acknowledgments}

This research receives support from the NNSF of China (Grant No. 10604051), the CAS, and the National Fundamental Research Program.

[1] A. S. Holevo, Probabilistic and Statistical Aspects of Quantum Theory (North-Holland Publishing Company, Amsterdam, 1982).

[2] A. S. Holevo, Statistical Structure of Quantum Theory (Springer-Verlag, Berliln, 2000).

[3] C. W. Helstrom, Quantum Detection and Estimation Theory (Academic Press, New York, 1976).

[4] W. K. Wooters, W. H. Zurek, Nature 299, 802 (1982).

[5] A. K. Pati and S. L. Braunstein, Nature 404, 164 (2000).

[6] W. H. Zurek, Nature 404, 130 (2000).

[7] A. S. Holevo, J. Multivariate Anal. 3, 337 (1973).

[8] H. P. Yuen, R. S. Kennedy, and M. Lax, IEEE Trans. Inf. Theory IT-21, 125 (1975).

[9] S. M. Barnett, Phys. Rev. A 64, 030303(R) (2001).

[10] C. -L. Chou, Phys. Rev. A 70, 062316 (2004).

[11] D. Qiu, Phys. Rev. A 77, 012328 (2008).

[12] M. Ježek, J. Řháček and J. Fiurášek, Phys. Rev. A 65, 060301(R) (2002).

[13] E. Andersson, S. M. Barnett, C. R. Gilson and K. Hunter, Phys. Rev. A 65, 052308 (2002).

[14] U. Herzog and J. A. Bergou, Phys. Rev. A 65, 050305(R) (2002).

[15] Y. C. Eldar, A. Megretski and G. C. Verghese, IEEE Trans. Inf. Theory 50, 1198 (2004).

[16] C. -L. Chou and L. Y. Hsu, Phys. Rev. A 68, 042305 (2003).

[17] V. P. Belavkin, Stochastics 1, 315 (1975).

[18] V. P. Belavkin, Radio Eng. Electron. Phys. 20, 39 (1975).

[19] V. P. Belavkin and A. G. Vancjan, Radio Eng. Electron. Phys. 19, 1391 (1974).

[20] A. S. Holevo, Theor. Probab. Appl. 23, 411 (1978).

[21] P. Hausladen and W. K. Wootters, J. Mod. Opt. 41, 2385 (1994).

[22] Y. C. Eldar and G. D. Forney, Jr., IEEE Trans. Inf. Theory 47, 858 (2001).

[23] J. Tyson, Phys. Rev. A 79, 032343 (2009).

[24] J. Tyson, J. Math. Phys. 50, 032106 (2009).

[25] K. Hunter, Phys. Rev. A 68, 012306 (2003).

[26] C. Mochon, Phys. Rev. A 73, 032328 (2006).

[27] I. D. Ivanovic, Phys. Lett. A 123, 257 (1987).

[28] D. Dieks, Phys. Lett. A 126, 303 (1988).

[29] A. Peres, Phys. Lett. A 128, 19 (1988).

[30] G. Jaeger and A. Shimony, Phys. Lett. A 197, 83 (1995).

[31] A. Chefles, Phys. Lett. A 239, 339 (1998).

[32] L. M. Duan and G. C. Guo, Phys. Rev. Lett. 80, 4999 (1998).

[33] A. Peres and D. R. Terno, J. Phys. A 31, 7105 (1998).

[34] Y. Sun, M. Hillery and J. A. Bergou, Phys. Rev. A 64, 022311 (2001).

[35] S. Zhang, Y. Feng, X. Sun and M. Ying, Phys. Rev. A 64, 062103 (2001).

[36] S. Zhang and M. Ying, Phys. Rev. A 65, 062322 (2002).

[37] Y. Sun, J. A. Bergou and M. Hillery, Phys. Rev. A 66, 032315 (2002).

[38] M. A. Jafarizadeh, M. Rezaei, N. Karimi and A. R. Amiri, Phys. Rev. A 77, 042314 (2008).

[39] A. Chefles and S. M. Barnett, Phys. Lett. A 250, 223 (1998).

[40] T. Rudolph, R. W. Spekkens and P. S. Turner, Phys. Rev. A 68, 010301(R) (2003).

[41] C. Zhang, G. Wang and M. Ying, Phys. Rev. A 75, 062306 (2007).

[42] U. Herzog and J. A. Bergou, Phys. Rev. A 71, 050301(R) (2005).

[43] P. Raynal and N. Lütkenhaus, Phys. Rev. A 76, 052322 (2007).

[44] U. Herzog, Phys. Rev. A 75, 052309 (2007).

[45] X. -F. Zhou, Y. -S. Zhang and G. -C. Guo, Phys. Rev. A 75, 052314 (2007)

[46] P. Raynal, N. Lütkenhaus and S. J. van Enk, Phys. Rev. A 68, 022308 (2003).

[47] Y. Feng, R. Duan and M. Ying, Phys. Rev. A 70, 012308 (2004).

[48] P. Raynal and N. Lütkenhaus, Phys. Rev. A 72, 022342 (2005).

[49] Y. C. Eldar, M. Stojnic and B. Hassibi, Phys. Rev. A 69, 062318 (2004).

[50] A. Chefles and S. M. Barnett, J. Mod. Opt. 45, 1295 (1998).

[51] C. -W. Zhang, C. -F. Li and G. -C. Guo, Phys. Lett. A 261, 25 (1999).

[52] M. A. P. Touzel, R. B. A. Adamson and A. M. Steinberg, Phys. Rev. A 76, 062314 (2007).

[53] J. Fiurášek and M. Ježek, Phys. Rev. A 67, 012321 (2003).

[54] A. S. Holevo, Probl. Peredachi Inf. 10, 51 (1974); A. S. Holevo, Probl. Inf. Transm. 10, 51 (1974). 
[55] Y. C. Eldar, A. Mergretski and G. C. Verghese, IEEE Trans. Inf. Theory 49, 1007 (2003).

[56] Y. C. Eldar, IEEE Trans. Inf. Theory 49, 446 (2003).

[57] A. Peres, Quantum Theory: Concepts and Methods (Kluwer Academic Publishers, Dordrecht, 1993).

[58] The Cauchy-Schwartz inequality: Let $\boldsymbol{x}$ and $\boldsymbol{y}$ be two arbitrary vectors in a Hilbert space, then $\|\boldsymbol{x} \cdot \boldsymbol{y}\| \leq \sqrt{\|\boldsymbol{x}\|^{\frac{1}{2}}\|\boldsymbol{y}\|^{\frac{1}{2}}}$, where $\|\cdot\|$ denotes the norm of a vector in the given Hilbert space, and the "=" holds if and only if $\boldsymbol{x}$ and $\boldsymbol{y}$ are linearly dependent.

[59] Roger A. Horn and Charles R. Johnson, Matrix Analysis, Vol. 1 (Cambridge University Press, Cambridge, 1986).

[60] Peter N. Brown and Youcef Saad, SIAM J. Sci. and Stat. Comput. 11, 450 (1990).

[61] J. E. Dennis, Jr. and Robert B. Schnabel, Numerical Methods for Unconstrained Optimization and Nonlinear Equations (Prentice Hall, New York, 1983).

[62] J. M. Ortega and W. C. Rheinboldt, Iterative Solution of Nonlinear Equations in Several Variables (Academic Press, New York and London, 1970).

[63] Alfio Quarteroni, Riccardo Sacco and Fausto Saleri, Numerical Mathematics (Springer-Verlag, Berlin, 2000).

[64] A stationary point of a function $F\left(x_{1}, \cdots, x_{n}\right)$ is a point in the domain of the function where $\frac{\partial F}{\partial x_{i}}=0, i=1, \cdots, n$.

[65] G. M. D'Ariano, M. F. Sacchi and J. Kahn, Phys. Rev. A 72, 032310 (2005).

[66] G. M. D'Ariano, M. F. Sacchi and J. Kahn, Phys. Rev. A 72, 052302 (2005).

[67] Sarah Croke, Erika Andersson, Stephen M. Barnett, Claire R. Gilson and John Jeffers, Phys. Rev. Lett. 96, 070401 (2006).

[68] Ulrike Herzog, Phys. Rev. A 79, 032323 (2009).

[69] Vieta's theorem characterizes the relation between the coefficients and the roots of a polynomial equation as follows: suppose $x^{n}+a_{1} x^{n-1} \cdots+a_{n-1} x+a_{n}=0$ is a polynomial equation defined on the complex field $\mathbb{C}$ and it has $n$ roots denoted by $x_{1}, \cdots, x_{n}$, then there must be $\sum_{1 \leq i_{1} \leq \cdots \leq i_{k} \leq n} x_{i_{1}} x_{i_{2}} \cdots x_{i_{k}}=(-1)^{k} a_{k}$.

[70] The mean inequality is $\frac{1}{n} \sum_{i=1}^{n} x_{i} \geq\left(\prod_{i=1}^{n} x_{i}\right)^{\frac{1}{n}}$ provided that $x_{1}, \cdots, x_{n}$ are $n$ positive real numbers. And the condtion that " $="$ holds in the mean inequality is $x_{1}=\cdots=x_{n}$. 